\title{
Diagnosing Childhood-onset Inborn Errors of Metabolism by Next Generation Sequencing
}

DOI:

10.1136/archdischild-2017-312738

\section{Document Version}

Accepted author manuscript

Link to publication record in Manchester Research Explorer

\section{Citation for published version (APA):}

Ghosh, A., Schlecht, H., Heptinstall, L., Bassett, J. K., Cartwright, E., Bhaskar, S., Urquhart, J., Broomfield, A., Morris, A. AM., Jameson, E., Schwahn, B. C., Walter, J., Douzgou, S., Murphy, H., Hendriksz, C., Sharma, R., Wilcox, G., Crushell, E., Monavari, A. A., ... Banka, S. (2017). Diagnosing Childhood-onset Inborn Errors of Metabolism by Next Generation Sequencing. Archives of Disease in Childhood.

https://doi.org/10.1136/archdischild-2017-312738

\section{Published in:}

Archives of Disease in Childhood

\section{Citing this paper}

Please note that where the full-text provided on Manchester Research Explorer is the Author Accepted Manuscript or Proof version this may differ from the final Published version. If citing, it is advised that you check and use the publisher's definitive version.

\section{General rights}

Copyright and moral rights for the publications made accessible in the Research Explorer are retained by the authors and/or other copyright owners and it is a condition of accessing publications that users recognise and abide by the legal requirements associated with these rights.

\section{Takedown policy}

If you believe that this document breaches copyright please refer to the University of Manchester's Takedown Procedures [http://man.ac.uk/04Y6Bo] or contact uml.scholarlycommunications@manchester.ac.uk providing relevant details, so we can investigate your claim.

\section{OPEN ACCESS}


Diagnosing Childhood-onset Inborn Errors of Metabolism by Next Generation

\section{Sequencing}

Arunabha Ghosh, Helene Schlecht, Lesley E Heptinstall, John K Bassett, Eleanor

Cartwright, Sanjeev Bhaskar, Jill Urquhart, Alexander Broomfield, Andrew AM

Morris, Elisabeth Jameson, Bernd C Schwahn, John H Walter, Sofia Douzgou, Helen

Murphy, Christian Hendriksz, Reena Sharma, Gisela Wilcox, Ellen Crushell, Ardeshir A

Monavari, Richard Martin, Anne Doolan, Senthil Senniappan, Simon C Ramsden,

Simon A Jones, Siddharth Banka

\begin{tabular}{|c|c|c|}
\hline Author name & Affiliation & Degree(s) \\
\hline Arunabha Ghosh & 1,2 & MA (cantab) MBBChir MSc MRCPCH \\
\hline Helene Schlecht & 1 & MSc, PhD \\
\hline Lesley E Heptinstall & 1 & BA MSc \\
\hline John K Bassett & 1 & BSc MBChB \\
\hline Eleanor Cartwright & 1 & $\mathrm{BSC}$ \\
\hline Sanjeev Bhaskar & 1 & BSc MSc \\
\hline Jill Urquhart & 1 & BSc (Hons), PhD \\
\hline Alexander Broomfield & 1 & MBBS MSc \\
\hline Andrew AM Morris & 1 & BM BCh MA PhD \\
\hline Elisabeth Jameson & 1 & MBBCh BSc MRCPCH \\
\hline Bernd C Schwahn & 1 & $\mathrm{MD}$ \\
\hline John H Walter & 1 & MD \\
\hline Sofia Douzgou & 1,2 & $\mathrm{MD}, \mathrm{MSc}, \mathrm{PhD}$ \\
\hline Helen Murphy & 1 & \\
\hline Christian Hendriksz & 3,8 & $\mathrm{MB}$ ChB, MSc, MRCP FRCPCH \\
\hline Reena Sharma & 3 & \\
\hline Gisela Wilcox & 3,9 & BMedSc(Hons) MBBS(Hons) MD FRACP MAACB FRCPA \\
\hline Ellen Crushell & 4 & MB BCh BAO, MRCPI(Paed), FFPAED (RCPI) \\
\hline Ardeshir A Monavari & 4 & MBChB.,DCH.,MRCP(UK).,RFCPCH.,FRCPIreland \\
\hline Richard Martin & 5 & \\
\hline Anne Doolan & 6 & MB BCh BAO BMedSc MD MRCPI \\
\hline Senthil Senniappan & 7 & MD FRCPCH MSc(Diab) PhD \\
\hline Simon C Ramsden & 1 & PhD, FRCPath \\
\hline Simon A Jones & 1 & $\mathrm{BSc}, \mathrm{MBChB}$ \\
\hline Siddharth Banka & 1,2 & MBBS MRCPCH PhD \\
\hline
\end{tabular}




\section{Affiliation list}

\begin{tabular}{|c|c|}
\hline 1 & $\begin{array}{l}\text { Manchester Centre for Genomic Medicine, St Mary's Hospital, Manchester } \\
\text { Academic Health Science Centre (MAHSC), Manchester, UK }\end{array}$ \\
\hline 2 & $\begin{array}{l}\text { Manchester Centre for Genomic Medicine, Institute of Human Development, } \\
\text { University of Manchester, Manchester, UK }\end{array}$ \\
\hline 3 & Mark Holland Metabolic Unit, Salford Royal Hospital, Salford, UK \\
\hline 4 & $\begin{array}{l}\text { National Centre for Inherited Metabolic Disorders, Temple Street Children's } \\
\text { University Hospital, Dublin, Ireland }\end{array}$ \\
\hline 5 & Institute of Human Genetics, The International Centre for Life, Newcastle, UK \\
\hline 6 & Cork University Maternity Hospital, Cork, Ireland \\
\hline 7 & Department of Endocrinology, Alder Hey Children's Hospital, Liverpool, UK \\
\hline 8 & $\begin{array}{l}\text { Department of Paediatrics and Child Health, Steve Biko Academic Unit, } \\
\text { University of Pretoria, South Africa }\end{array}$ \\
\hline 9 & $\begin{array}{l}\text { School of Clinical Sciences, Monash University, Clayton, Victoria } 3145 \\
\text { Australia }\end{array}$ \\
\hline
\end{tabular}

\section{CORRESPONDENCE}

Dr Siddharth Banka

Manchester Centre for Genomic Medicine

University of Manchester

St. Mary's Hospital

Oxford Road

Manchester. M13 9WL. U.K.

Tel: $+44(0) 1617010980$

Fax: $+44(0) 1612766145$

Email: siddharth.banka@manchester.ac.uk

\section{WORD COUNT}

3242 (not including Abstract, legends, references) 


\section{ABSTRACT}

Background: Inborn errors of metabolism (IEMs) underlie a substantial proportion of paediatric disease burden but their genetic diagnosis can be challenging using the traditional approaches.

Methods: We designed and validated a Next Generation Sequencing (NGS) panel of 226 IEM genes, created six overlapping phenotype-based sub-panels and tested 102 individuals, who presented clinically with suspected childhood-onset IEMs.

Results: In 51/102 individuals, NGS fully or partially established the molecular cause or identified other actionable diagnoses. Causal mutations were identified significantly more frequently when the biochemical phenotype suggested a specific IEM or a group of IEMs $(p<0.0001)$, demonstrating the pivotal role of prior biochemical testing in guiding NGS analysis. The NGS panel helped to avoid further invasive, hazardous, lengthy or expensive investigations in $69 \%$ individuals $(p<0 \cdot 0001)$. Additional functional testing due to novel or unexpected findings had to be undertaken in only $3 \%$ of subjects, demonstrating that use of NGS does not significantly increase the burden of subsequent follow-up testing. Even where a molecular diagnosis could not be achieved, NGS-based approach assisted in the management and counselling by reducing the likelihood of a high-penetrant genetic cause.

Conclusions: NGS has significant clinical utility for the diagnosis of IEMs. Biochemical testing and NGS analysis play complementary roles in the diagnosis of IEMs. Incorporating NGS into the diagnostic algorithm of IEMs can improve the accuracy of diagnosis.

\section{KEY WORDS}

Inborn errors of metabolism; metabolic disorders; next generation sequencing 


\section{MAIN TEXT}

Inborn errors of metabolism (IEMs) result in a range of childhood- or adult-onset phenotypes and are caused by the disruption of biochemical pathways. IEMs occur in 1 in 800 to 2500 births [1 2]. Metabolic investigations are an integral part of their diagnostic process [3 4] and are essential for enabling management that may prevent disability, limit disease or be curative [5-9]. IEM investigations can be broadly divided in two overlapping categories - (i) screening tests (newborn screening or phenotype-directed) to detect abnormal biochemical markers (e.g. acylcarnitine profile, organic acid profile in urine, or amino acid profiles in plasma/urine) or (ii) specific assays to detect precise deficiencies (e.g. specific pathognomonic biomarkers, cellular complementation or enzyme activity assays) [10]. However, some IEMs lack reliable biochemical markers or if present, the relevant investigations can be invasive, prohibitively expensive, time consuming or offered only on an ad hoc basis by research laboratories. Even for IEMs with accessible biochemical markers, genetic testing is often needed to improve the accuracy of diagnosis, determine prognosis, for cascade carrier testing and prenatal or pre-implantation diagnosis. In the era of personalised medicine, genetic testing will facilitate emerging genotype-specific therapies [11].

Next Generation Sequencing (NGS) has changed the diagnostic paradigm of rare genetic disorders [12-17]. Diagnostic yield is a key parameter of the clinical utility of NGS [18]. Systematic evaluation of diagnostic yields of NGS across different disease groups will help in the rational integration of NGS into clinical practice. IEMs are potentially excellent candidates for an NGS-based approach due to their extreme genetic heterogeneity and complexities of the traditional diagnostic approach. 
However, the role of NGS in the diagnostic algorithm of IEMs and its broader clinical impact remain to be established. Here we report 102 individuals who underwent NGS for suspected childhood-onset IEM in a clinical diagnostic setting.

\section{Methods and Results}

\section{Panel design and sequencing}

A list of over 500 known IEM genes was prepared in April 2014 [19]

(www.omim.org). From this list we excluded mitochondrial disorders and congenital disorders of glycosylation because their genetic heterogeneity remains substantially unresolved (Supplementary Table S1). This makes whole exome (WES) or whole genome sequencing (WGS) more appropriate for these two groups. Finally, we selected 226 IEM genes with predominantly neurodevelopmental phenotypes that are genetically heterogeneous, or are diagnosed through specialist investigations which are invasive, expensive or have poor availability. We created overlapping subpanels based on biochemical and clinical phenotypes to optimise the bioinformatic analysis and minimise secondary findings. The sub-panels were - (i) amino acid and neurotransmitter defects; (ii) organic acidaemias and vitamins-related disorders; (iii) disorders of fatty acid oxidation or ketone metabolism and hyperammonaemia; (iv) carbohydrate metabolism defects; (v) lysosomal disorders and neuronal ceroid lipofuscinoses and (vi) peroxisomal disorders. A full list of the selected genes and the sub-panels is provided in Table S2.

We designed a targeted enrichment to sequence all the exons (+/-50 base pairs), of the canonical transcripts of the selected 226 genes. Additionally, we included all the published pathogenic intronic variants for the selected genes (information taken 
from http://www.hgmd.cf.ac.uk). Using manufacturers' protocols, DNA samples from peripheral blood were enriched by an Agilent SureSelect Custom Design targetenrichment kit (Agilent, Santa Clara, CA, USA) and sequenced with the Illumina HiSeq 2500 (Illumina, Inc., San Diego, CA, USA). Subsequent sequence alignment, variant calling and annotation and filtering were performed as reported previously[20]. If a heterozygous variant in a clinically relevant recessive-disorder-linked gene was identified, then ExomeDepth program [21] was used to identify call copy number variants (CNVs) in the relevant gene.

Sensitivity of the assay was determined by comparing calls from four cell lines, with published genotypes, derived from The International HapMap Project and the 1000 Genomes Project (NA19194, HG01970, NA19005, NA18907; Corriell Institute, 403 Haddon Avenue Camden, New Jersey, 08103, USA). In total, 1216 known single nucleotide variants, within the region of interest, were cross-referenced with the locally generated NGS data. This analysis did not identify any false-positive or falsenegative calls. We undertook NGS runs on four individuals with previously known mutations in at least one of the 226 selected genes and confirmed the ability of the assay to identify the expected mutations.

A repeat independent enrichment and sequencing run on one of the four cell lines was undertaken and results compared to test assay-repeatability, which demonstrated $100 \%$ match with original run along with comparable coverage for the region of interest. In summary, we designed and validated a robust targeted NGS analysis assay for a comprehensive range of IEMs, that has a minimum 50X coverage for $>97 \%$ bases and a specificity of $100 \%$ and sensitivity of $100 \%$ within exons $+/-5$ 
bases. No known areas in the 226 selected genes consistently failed to achieve this coverage.

\section{Clinical data collection, reporting and statistical analysis}

This work is a retrospective evaluation of a clinical diagnostic service and ethical approval was not required. Samples along with completed clinical proformas (Supplementary Information) were accepted from clinicians for analysis of either a single sub-panel or any combination of sub-panels. Clinical and biochemical profiling of most patients was undertaken prior to the referral for NGS analysis by IEM specialists or geneticists from tertiary centres (Table 1, Table S3). NGS panel analysis was performed for 102 individuals with suspected IEMs of childhood-onset.

A multi-disciplinary team (MDT) comprising of clinical scientists, clinical geneticists, IEM specialists and genetic counsellors was constituted for clinical correlation of results. Findings were reported under three categories (i) primary - likely clinically significant pathogenic variants; (ii) secondary - deleterious but likely incidental carrier findings; and (iii) variants of unknown clinical significance (VUS) - variants with insufficient population frequency data to evaluate their deleteriousness. All primary and secondary point mutations were confirmed with bi-directional Sanger sequencing and CNVs were confirmed by dosage assays. Where relevant and possible, parental segregation studies were performed.

Primary pathogenic variants were reported in 51/102 individuals (Table 1). In 51/102 no primary pathogenic variants were detected (Table S3). In 18/102 (8 with and 10 without primary pathogenic findings) secondary pathogenic carrier variants were 
reported (Table S4). In total, 89 pathogenic variants were identified across 58/226 genes in 61 individuals. Of these, $27 / 89$ (30\%) were novel and the remainder have been previously reported in the literature as pathogenic variants.

For overall analysis of distribution of diagnostic yields and carrier frequencies in this study, the data were grouped according to the complexity of the presenting phenotype and number of sub-panels requested. $\chi^{2}$ tests were performed using IBM SPSS Statistics v24. Results of these analyses are summarised in Figure 1.

\section{Discussion}

The relative merits and limitations of WGS, WES and custom targeted-design have been discussed in the literature [12 14-17 20 22-24]. We chose bespoke targeted enrichment to guarantee a uniformly high coverage of the regions of interest. Our approach allowed for the inclusion of known intronic mutations that would escape detection via exome sequencing. Notably, we identified a deep-intronic PTS c.84323A > T mutation in subject \#30 (Table 1) with tetrahydrobiopterin-deficient hyperphenylalaninemia (OMIM 261640).

Our cohort of 102 individuals includes five adults, three individuals with known onset of disease in childhood and two parents (subjects \#11 and \#12) of a sibship of three deceased children with suspected GM1 gangliosidosis (OMIM 230500). In 42/51 individuals the reported primary pathogenic variant was fully consistent with their biochemical and clinical features (Table 1). In a further 7/51 individuals, heterozygous pathogenic variants were detected in relevant recessive disorder genes (subjects $\# 3,6,8,15,27,42,50$ ). These may be incidental carrier findings or 
partial diagnoses where a second variant escaped detection. In 2/51 individuals the genetic diagnosis did not fully explain the presenting phenotype (Tyrosinaemia type I, OMIM 276700 in subject \#47 and peroxisomal acyl-CoA oxidase deficiency, OMIM 264470 in subject \#51). Incidental additional diagnoses with clear management implications were made in two individuals with other confirmed or possible diagnoses (Fabry disease, OMIM 301500 in subject \#11 and galactokinase deficiency, OMIM 230200 in subject \#51). Overall, the panel provided clinically useful and actionable information in half $(51 / 102)$ of the individuals tested.

Identification of causal mutations had direct clinical impact on a number of families and individuals (Table 1). We diagnosed an ultra-rare condition in subject \#41 (HMGCoA synthase-2 deficiency, OMIM 605911) that could not have been identified just on clinical or biochemical features. Diagnosis of glycogen storage disorder 0 (OMIM 240600) in subject \#43 led to a change in management strategy from frequent bolus feeds to regular overnight feeds, necessitating gastrostomy insertion. Accurate genetic diagnosis enabled targeted cascade genetic testing for a number of relatives and subsequently led to diagnostic confirmation in three siblings. Five prenatal tests were offered following NGS results and one fetus was confirmed to be affected. Although we have not undertaken a formal cost-benefit analysis, the cost of NGS was comparable with the traditional diagnostic approach in mutation positive cases (Table 1) (Table S7).

When clinical or biochemical features suggested a specific single gene defect or a particular sub-group of IEMs, NGS analysis led to a confirmed diagnosis in 39/66 
(59\%) individuals, whereas when an IEM was suspected but clinical and biochemical features were nonspecific, genetic diagnoses were made in only $3 / 36$ by this approach $(8 \%)(p<0.0001)$. This underlines the role of biochemical investigations and phenotyping in directing NGS data analysis.

The likelihood of confirming a genetic diagnosis in individuals with a suspected specific single gene defect was high (24/34, 71\%). Admittedly, a panel-based approach in such cases appears counterintuitive. However, targeted clinical sequencing is not available in the UK for $16 / 34$ suspected single gene defects.. improves access to genetic testing for IEMs.

Certain biochemical markers, such as very long chain fatty acids (VLCFA, indicative of peroxisomal disorders), methylmalonic aciduria (MMA, indicative of methylmalonic acidaemia or cobalamin metabolism defects) or specific enzyme deficiencies, led to better diagnostic yields than markers such as isolated hypoglycaemia or hyperammonaemia (Figure 1B). Our results suggest that for certain groups of disorders, NGS can be reliable, cost-effective, quicker and less invasive than followup biochemical testing. For example, confirmatory biochemical diagnosis of specific peroxisomal disorders, MMA or cobalamin metabolism defects usually requires functional testing in cultured fibroblasts, necessitating a skin biopsy followed by lengthy highly specialist complementation or enzyme studies [25 26].

The diagnostic yield of NGS for hypoglycaemia and hyperammonaemia was comparatively lower than for VLCFA or MMA (Figure 1B), but consistent with the known positive predictive value of these markers [27 28]. For hypoglycaemia, the 
NGS diagnostic yield (27\%) is comparable to that of diagnostic fasting (22\%) [27].

Fasting requires hospital admission and is potentially hazardous, though it may still have a role in optimising management [29]. For hyperammonaemia, the NGS yield (45\%) is comparable to that of standard confirmatory tests (54\%) [28]. For both hypoglycaemia and hyperammonaemia, the yield improved in the presence of an additional biochemical marker (Figure 1B).

There were 23 incidences of carrier findings across 21 genes in 18/102 (17\%) individuals (Table S4). By dividing the assay into sub-panels we minimised incidental carrier findings. VUS were detected in 85/102 (83\%) of individuals, with a median of 3 VUS detected per individual (range 0-24) (Table S5). These results reflect the importance of pre-test counselling and consent for NGS-based tests.

Scientific analysis of variants to determine the pathogenicity is challenging and compounded by the large number of variants identified in multi-gene NGS analyses. We identified 89 pathogenic mutations across 58 genes, thus expanding the mutational spectrum for these genes. If there was insufficient prior evidence to confirm the significance of the detected genetic variant we undertook reverse phenotyping using clinical, biochemical or in silico approaches (e.g. subject \#41 with HMG-CoA synthase-2 deficiency, OMIM 605911) [30]. Interestingly, even though $27 / 89$ pathogenic variants were novel, the burden of additional functional testing due to NGS results was minimal and had to be considered in only $3 \%$ of subjects (Table 1). In most cases, review of previously performed biochemical tests was sufficient for correlation of unexpected NGS findings. In comparison, the NGS approach helped to avoid invasive, hazardous, lengthy or expensive investigations in 
$69 \%$ (Tables 1 and S2). This demonstrates the robustness of available variant and mutation databases and in silico tools for this group of disorders and underscores the clinical utility of this approach.

A proportion of the 51/102 individuals (Table S3) in whom we failed to achieve a genetic diagnosis may have mutations in genes that are not part of our panel. In addition, certain classes of mutations may have escaped detection due to technical limitations of the assay. Additionally, certain biochemical phenotypes such as recurrent hypoglycaemia or hyperammonaemia may be multi-factorial in origin in some patients, making it challenging to achieve precise genetic diagnoses.

Notably, using NGS is clinically useful even in cases where a molecular diagnosis is not achieved because a 'negative' NGS result can substantially reduce the likelihood of a high-penetrant single gene disorder and thus informing the management of the patient and counselling of the families. Furthermore, NGS was the only practical approach in several cases or helped to avoid inconvenient or invasive investigations. Even in these 'negative' cases, the cost of NGS was comparable to the investigations that would otherwise have been performed (Table S6). These individuals could form a focussed cohort for future WES or WGS research studies.

Although the potential for NGS in a few selected groups of IEMs has been previously reported, the use of NGS for multiple groups of IEMs has not been studied extensively [15 31-39] (summarised in Figure S1). The results presented here establish the utility of NGS for diagnosis of multiple groups of IEMs in clinical practice. Figure 2 illustrates how the use of NGS may be incorporated into the 
diagnostic algorithm of IEMs. We have demonstrated that biochemical tests are pivotal for the diagnosis of IEMs as they help focus genetic testing and facilitate NGS analysis. In addition, we have demonstrated that NGS can help to avoid invasive, expensive or hazardous biochemical confirmatory studies in specific situations, can improve access to genetic testing and may be a comparable approach in cost terms to alternative investigations. Overall, incorporating NGS into the diagnostic algorithm of IEMs can improve the accuracy of diagnosis. Further work is required to formally assess the cost-effectiveness of NGS and explore the optimal approach to the timing of NGS in the diagnosis of IEMs.

The future of genomic techniques in the diagnosis of IEMs is likely to involve WES and WGS, the latter conferring particular advantages including the ability to provide equal coverage across the genome, detect copy number variants, indels, intronic and regulatory variants. However, the pivotal role of biochemical testing as we demonstrate here and the potential ability of genomic testing to reduce the need for additional studies will remain relevant to WES and WGS. 
What is already known on this topic ( 3 statements, max 25 words per statement)

Next generation sequencing has changed the diagnostic paradigm for a number of rare genetic disorders.

Genetic testing in inborn errors of metabolism is important for accuracy of diagnosis, to provide information on prognosis, for cascade carrier testing and for prenatal or pre-implantation diagnosis.

Though research based NGS testing for IEM has been developed, the place of NGS in a clinical diagnostic setting has not been established.

What this study adds ( 3 statements, max 25 words per statement)

NGS based testing can improve the accuracy of diagnosis of IEM and potentially avoid the need for invasive or expensive functional testing.

Biochemical testing and NGS are complementary in the diagnosis of IEM.

NGS testing in patients with suspected IEM can significantly reduce the need for other invasive and expensive investigations. 
TABLE 1: Primary pathogenic variants from IEM NGS panel analysis for individuals with childhood-onset disease.

Results are grouped by sub-panels - (AMN, FAOD, KET), (LSD, NCL), (OA, VIT), (AA, NT), (CHO), (PER), followed by combinations.

Key - AA Disorders of amino acid metabolism including phenylketonuria, and cerebral organic acid disorders; AMN Disorders associated with hyperammonaemia; $\mathrm{CHO}$ Disorders of carbohydrate metabolism; FAOD Fatty acid oxidation defects including multiple acyl-CoA dehydrogenase deficiency; KET Disorders of ketogenesis or ketolysis; LSD Lysosomal disorders: includes lysosomal storage disorders, transport defects and protease defects; NCL Neuronal ceroid lipofuscinoses; NT Disorders of neurotransmission (includes pterins, tyrosinaemia); OA Organic acidaemias, including disorders of branched chain amino acid catabolism, 3-methylglutaconic acidurias; PER Peroxisomal biogenesis disorders and disorders of single peroxisomal enzymes; VIT Folate and cobalamin defects, also riboflavin transport defects, and biotin-responsive disorders.

Het heterozygous, hom homozygous, hemi hemizygous comp het compound heterozygous.

$\wedge$ Denotes invasive or hazardous or lengthy or expensive investigations that were avoided by using the NGS approach.

*Three SUMF1 variants were identified. Of the two cis variants (c.776A $>$ T p.(Asn25911e), c.797C >T p.(Pro266Leu)), (c.776A>T p.(Asn25911e) is considered to be the more pathogenic.[40] **Previously reported synonymous change resulting in a splicing defect.[41]

\begin{tabular}{|c|c|c|c|c|c|c|c|}
\hline $\begin{array}{l}\text { Subject } \\
\#\end{array}$ & $\begin{array}{l}\text { Approximate } \\
\text { age at } \\
\text { investigation }\end{array}$ & Key biochemical and clinical features & Result & Zygosity & $\begin{array}{l}\text { Interpretation and clinical } \\
\text { impact }\end{array}$ & Investigations avoided by NGS & $\begin{array}{l}\text { Investigations } \\
\text { added by NGS }\end{array}$ \\
\hline \multicolumn{8}{|c|}{ Single sub-panel tested: (AMN, FAOD, KET) } \\
\hline 1 & $<1$ year & $\begin{array}{l}\text { Neonatal encephalopathy, severe } \\
\text { hyperammonaemia, low arginine. Suspected } \\
\text { argininosuccinic aciduria. }\end{array}$ & $\begin{array}{l}\text { ASL c.749T>A } \\
{[p .(\text { Met250Lys)] }}\end{array}$ & hom & $\begin{array}{l}\text { Diagnosis of argininosuccinic } \\
\text { aciduria confirmed }\end{array}$ & $\begin{array}{l}\text { Single gene mutation analysis or ^enzyme } \\
\text { analysis in red blood cells followed by single } \\
\text { gene analysis }\end{array}$ & None \\
\hline 2 & $<1$ year & $\begin{array}{l}\text { Encephalopathy, hypertonia. } \\
\text { Hyperammonaemia with elevated citrulline. } \\
\text { Suspected citrullinaemia. }\end{array}$ & $\begin{array}{l}\text { ASS1 c.970+5G>A, c.892delG } \\
\text { [p.(?), (Glu298ArgfsTer18)] }\end{array}$ & comp het & $\begin{array}{l}\text { Diagnosis of citrullinaemia } \\
\text { confirmed }\end{array}$ & $\begin{array}{l}\text { Single gene mutation analysis } \\
\text { Cascade testing of relatives undertaken }\end{array}$ & None \\
\hline 3 & $1-11$ years & $\begin{array}{l}\text { Motor delay, muscle weakness during illness, } \\
\text { memory and concentration deficits. Elevated } \\
\text { medium chain acylcarnitines. Suspected Multiple } \\
\text { acyl-CoA dehydrogenase deficiency (MADD). }\end{array}$ & $\begin{array}{l}\text { ETFDH c.5ldupT } \\
\text { [p.(Ala18CysfsTer5)] }\end{array}$ & het & $\begin{array}{l}\text { Possible diagnosis of MADD } \\
\text { (no second variant found) }\end{array}$ & $\begin{array}{l}\text { ^Sequential mutation analysis of multiple } \\
\text { genes }\end{array}$ & None \\
\hline 4 & $1-11$ years & $\begin{array}{l}\text { Severe progressive myopathy with } \\
\text { myoglobinuria and hepatic steatosis, recurrent }\end{array}$ & $\begin{array}{l}\text { ETFDH c.413T>G } \\
\text { [p.(Leu138Arg)], c.1333T>C }\end{array}$ & comp het & Diagnosis of MADD confirmed & ^Sequential mutation analysis of multiple & None \\
\hline
\end{tabular}




\begin{tabular}{|c|c|c|c|c|c|c|c|}
\hline & & $\begin{array}{l}\text { hypoglycaemia. Elevated acylcarnitines, urine } \\
\text { hexanoylglycine and lactate. Suspected Multiple } \\
\text { acyl-CoA dehydrogenase deficiency (MADD). }\end{array}$ & [p.(Trp445Arg)] & & & $\begin{array}{l}\text { genes } \\
\text { Cascade testing of relatives undertaken }\end{array}$ & \\
\hline 5 & $<1$ year & $\begin{array}{l}\text { Hypoglycaemia, biochemical tests suggestive of } \\
\text { CPT II deficiency. Hypertrophic cardiomyopathy, } \\
\text { abnormal renal cortex and IUGR. }\end{array}$ & $\begin{array}{l}\text { CPT2 c.1838G >A } \\
\text { [p.(Gly613Glu)] }\end{array}$ & hom & $\begin{array}{l}\text { Diagnosis of CPT II deficiency } \\
\text { confirmed. } \\
\text { Prenatal testing undertaken, } \\
\text { fetus affected }\end{array}$ & $\begin{array}{l}\text { Single gene mutation analysis and/or } \\
\text { ^enzyme activity in muscle tissue or } \\
\text { fibroblasts }\end{array}$ & None \\
\hline 6 & $1-11$ years & $\begin{array}{l}\text { Recurrent hypoglycaemia, minimal ketonuria, } \\
\text { previously abnormal C14:1 carnitine. Possible } \\
\text { very long chain Acyl-CoA dehydrogenase } \\
\text { (VLCAD) deficiency }\end{array}$ & $\begin{array}{l}\text { ACADVL c.1591C>T sub- } \\
\text { [p.(Arg531Trp)] }\end{array}$ & het & $\begin{array}{l}\text { Carrier finding or possible } \\
\text { diagnosis of VLCAD deficiency } \\
\text { (but no second variant found) }\end{array}$ & Single gene mutation analysis & None \\
\hline 7 & $1-11$ years & $\begin{array}{l}\text { Haematological abnormalities, persistently } \\
\text { raised lysine in urine and plasma. Suspected } \\
\text { lysinuric protein intolerance }\end{array}$ & $S L C 7 A 7$ c. $625+1 \mathrm{G}>\mathrm{A}$ & hom & $\begin{array}{l}\text { Diagnosis of Lysinuric protein } \\
\text { intolerance achieved }\end{array}$ & Single gene mutation analysis & None \\
\hline 8 & $1-11$ years & $\begin{array}{l}\text { Reye-like illness. Hypoglycaemia, } \\
\text { hyperammonaemia, elevated lactate and ALT. } \\
\text { Suspected FAOD. }\end{array}$ & $\begin{array}{l}\text { ACADVL c.631 G>A } \\
\text { [p.(Gly211Ser)] }\end{array}$ & het & $\begin{array}{l}\text { Carrier finding or possible } \\
\text { diagnosis of VLCAD deficiency } \\
\text { (but no second variant found) }\end{array}$ & $\begin{array}{l}\wedge \text { Sequential mutation analysis of multiple } \\
\text { genes }\end{array}$ & None \\
\hline 9 & $1-11$ years & $\begin{array}{l}\text { Profound learning disability, inflammatory bowel } \\
\text { disease, biochemical diagnosis of citrullinaemia }\end{array}$ & $\begin{array}{l}\text { ASS1 c.1168G>A } \\
\text { [p.(Gly390Arg)] }\end{array}$ & hom & $\begin{array}{l}\text { Diagnosis of citrullinaemia } \\
\text { confirmed }\end{array}$ & Single gene mutation analysis & None \\
\hline 10 & $1-11$ years & $\begin{array}{l}\text { Recurrent myositis with raised creatine kinase. } \\
\text { Acylcarnitine profile suggests FAOD, possible } \\
\text { CPT II deficiency. }\end{array}$ & $\begin{array}{l}\text { CPT2 c.338C>T } \\
{[p .(\text { Ser113Leu)] }}\end{array}$ & hom & $\begin{array}{l}\text { Diagnosis of CPT II deficiency } \\
\text { confirmed }\end{array}$ & Single gene mutation analysis & None \\
\hline \multicolumn{8}{|c|}{ Single sub-panel tested: (LSD, NCL) } \\
\hline 11 & $\begin{array}{l}\text { Adult }(18+ \\
\text { years) }\end{array}$ & $\begin{array}{l}\text { Parent of three decreased children with } \\
\text { suspected GM1 gangliosidosis }\end{array}$ & $\begin{array}{l}\text { GLB1 c.75+2dupT } \\
\text { GLA c.1153A>G } \\
\text { [p.(Thr385Ala)] }\end{array}$ & 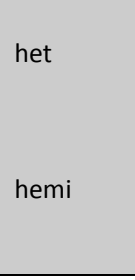 & 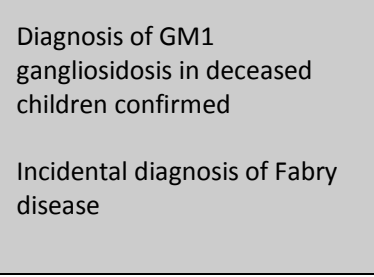 & $\begin{array}{l}\text { Single gene mutation analysis } \\
\text { None }\end{array}$ & $\begin{array}{l}\text { None } \\
\text { Enzyme assay in } \\
\text { leucocytes (for } \\
\text { confirmation of } \\
\text { Fabry disease) }\end{array}$ \\
\hline 12 & $\begin{array}{l}\text { Adult }(18+ \\
\text { years) }\end{array}$ & $\begin{array}{l}\text { Parent of three decreased children with } \\
\text { suspected GM1 gangliosidosis }\end{array}$ & GLB1 c. $75+2$ dupT & het & $\begin{array}{l}\text { Diagnosis of GM1 } \\
\text { gangliosidosis in deceased } \\
\text { children confirmed }\end{array}$ & Single gene mutation analysis & None \\
\hline 13 & $1-11$ years & $\begin{array}{l}\text { Global developmental delay. Elevated urine } \\
\text { GAGs and reduced activity of multiple lysosomal } \\
\text { sulfatases. Suspected multiple sulfatase }\end{array}$ & $\begin{array}{l}\text { SUMF1 c.776A>T } \\
\text { [p.(Asn259Ile)], c.797C>T } \\
\text { [p.(Pro266Leu)], c.836C }>T \text {, }\end{array}$ & comp het & $\begin{array}{l}\text { Diagnosis of multiple sulfatase } \\
\text { deficiency achieved. }\end{array}$ & Single gene mutation analysis & None \\
\hline
\end{tabular}




\begin{tabular}{|c|c|c|c|c|c|c|c|}
\hline & & deficiency. & [p.(Ala279Val)]* & & & & \\
\hline 14 & $<1$ year & Suspected galactosialidosis & $\begin{array}{l}\text { CTSA c.1284delG } \\
\text { [p.(Trp428CysfsTer18)] }\end{array}$ & hom & $\begin{array}{l}\text { Diagnosis of galactosialidosis } \\
\text { confirmed }\end{array}$ & $\begin{array}{l}\text { Skin biopsy, fibroblast culture, enzyme } \\
\text { activity in fibroblasts and single gene } \\
\text { mutation analysis }\end{array}$ & None \\
\hline 15 & $<1$ year & $\begin{array}{l}\text { Biochemical diagnosis of suspected } \\
\text { mucolipidosis type III. One GNPTAB mutation } \\
\text { already known. }\end{array}$ & $\begin{array}{l}\text { GNPTAB c.3503_3504delTC } \\
\text { [p.(Leu1168GInfsTer5)] }\end{array}$ & het & $\begin{array}{l}\text { Possible diagnosis of } \\
\text { mucolipidosis type III } \\
\text { alpha/beta (no second variant } \\
\text { found) }\end{array}$ & None, one mutation already known & None \\
\hline 16 & $1-11$ years & $\begin{array}{l}\text { Lower limb spasticity, deteriorating vision, loss } \\
\text { of speech. Reduced arylsulfatase A activity. } \\
\text { Suspected metachromatic leukodystrophy }\end{array}$ & $A R S A$ c. $465+1 \mathrm{G}>\mathrm{A}$ & hom & $\begin{array}{l}\text { Diagnosis of metachromatic } \\
\text { leukodystrophy confirmed. } \\
\text { Prenatal testing undertaken - } \\
\text { fetus unaffected }\end{array}$ & $\begin{array}{l}\text { Single gene mutation analysis, urinary } \\
\text { sulfatides }\end{array}$ & None \\
\hline 17 & $12-17$ years & $\begin{array}{l}\text { Biochemical (enzyme) diagnosis of mucolipidosis } \\
\text { type III, no mutations found in GNPTAB }\end{array}$ & $\begin{array}{l}\text { GNPTG c.196C>T } \\
\text { [p.(Arg66Ter)] }\end{array}$ & hom & $\begin{array}{l}\text { Diagnosis of Mucolipidosis III } \\
\text { gamma achieved } \\
\text { Genetic testing of sibling } \\
\text { undertaken and found to be } \\
\text { unaffected. }\end{array}$ & $\begin{array}{l}\text { ^Sequential mutation analysis of multiple } \\
\text { genes } \\
\text { Cascade testing of relatives undertaken }\end{array}$ & None \\
\hline 18 & $1-11$ years & $\begin{array}{l}\text { Developmental delay, behavioural disturbance. } \\
\text { Elevated urine heparan sulfate with deficient } \\
\text { HGSNAT activity in leukocytes, biochemical } \\
\text { diagnosis of MPS IIIC. }\end{array}$ & $\begin{array}{l}\text { HGSNAT c.947G>A } \\
\text { [p.(Trp316Ter)], c.1464+1 } \\
\text { G>A }\end{array}$ & comp het & $\begin{array}{l}\text { Diagnosis of } \\
\text { Mucopolysaccharidosis type } \\
\text { IIIC (Sanfilippo C) confirmed }\end{array}$ & Single gene mutation analysis & None \\
\hline 19 & $<1$ year & $\begin{array}{l}\text { Failure to thrive, hepatomegaly. Biochemical } \\
\text { diagnosis of Wolman disease. }\end{array}$ & LIPA (whole gene deletion) & hom & $\begin{array}{l}\text { Diagnosis Wolman disease } \\
\text { confirmed. }\end{array}$ & Single gene mutation analysis & None \\
\hline \multicolumn{8}{|c|}{ Single sub-panel tested: (OA, VIT) } \\
\hline 20 & $1-11$ years & $\begin{array}{l}\text { Vomiting and respiratory distress. Metabolic } \\
\text { acidosis with elevated methylmalonic acid in } \\
\text { urine and blood. Biochemical diagnosis of } \\
\text { methylmalonic acidaemia. }\end{array}$ & $\begin{array}{l}\text { MMAA c.433C }>T \\
{[p .(\operatorname{Arg} 145 T e r)]}\end{array}$ & hom & $\begin{array}{l}\text { Diagnosis of cblA type } \\
\text { methylmalonic aciduria } \\
\text { achieved }\end{array}$ & $\begin{array}{l}\text { ^Skin biopsy, fibroblast culture, } \\
\text { complementation assays and sequencing of } \\
\text { specific gene, or sequential mutation } \\
\text { analysis of multiple genes }\end{array}$ & None \\
\hline 21 & $1-11$ years & $\begin{array}{l}\text { Vomiting and weight loss, with macrocytic } \\
\text { anaemia and severe vitamin B12 deficiency. } \\
\text { Suspected cobalamin transport abnormality. }\end{array}$ & $\begin{array}{l}\text { GIF c. } 1155 \mathrm{C}>\mathrm{A} \\
\text { [p.(Tyr385Ter)], c.685G>A } \\
\text { [p.(Ala229Thr)] }\end{array}$ & comp het & $\begin{array}{l}\text { Diagnosis of Intrinsic Factor } \\
\text { deficiency achieved. Genetic } \\
\text { testing of sibling undertaken } \\
\text { and found to be affected. }\end{array}$ & $\begin{array}{l}\wedge \text { Sequential analysis of multiple genes } \\
\text { and/or Schilling test }\end{array}$ & None \\
\hline 22 & $1-11$ years & $\begin{array}{l}\text { Encephalopathy and cognitive deficit with } \\
\text { elevated homocysteine and urinary } \\
\text { methylmalonic acid. Suspected cblC disease. }\end{array}$ & $\begin{array}{l}\text { MMACHC c.394C>T } \\
\text { [p.(Arg132Ter)] }\end{array}$ & hom & $\begin{array}{l}\text { Diagnosis of cblC type } \\
\text { methylmalonic aciduria and } \\
\text { homocystinuria, confirmed. } \\
\text { Genetic testing of sibling } \\
\end{array}$ & $\begin{array}{l}\text { ^Skin biopsy, fibroblast culture, } \\
\text { complementation assays and sequencing of } \\
\text { specific gene, or sequential mutation }\end{array}$ & None \\
\hline
\end{tabular}




\begin{tabular}{|c|c|c|c|c|c|c|c|}
\hline & & & & & $\begin{array}{l}\text { undertaken and found to be } \\
\text { affected. }\end{array}$ & analysis of multiple genes & \\
\hline 23 & $<1$ year & $\begin{array}{l}\text { Elevated methylmalonic acid in urine. Suspected } \\
\text { methylmalonic acidaemia. }\end{array}$ & $\begin{array}{l}\text { MUT c.1207C>T } \\
{[\text { [p. (Arg403Ter)], c.52C>T }} \\
{[p .(\text { Gln18Ter)] }}\end{array}$ & comp het & $\begin{array}{l}\text { Diagnosis of mut }(0) \text { type } \\
\text { methylmalonic acidaemia } \\
\text { achieved. }\end{array}$ & $\begin{array}{l}\text { ^Skin biopsy, fibroblast culture, } \\
\text { complementation assays and sequencing of } \\
\text { specific gene, or sequential mutation } \\
\text { analysis of multiple genes }\end{array}$ & None \\
\hline 24 & $1-11$ years & $\begin{array}{l}\text { Developmental delay with myoclonic-astatic } \\
\text { epilepsy. Elevated homocysteine, normal } \\
\text { methionine, no MMA. }\end{array}$ & $\begin{array}{l}\text { MTHFR c. } 1129 C>T \\
{[p .(\operatorname{Arg} 377 \text { Cys })]}\end{array}$ & hom & $\begin{array}{l}\text { Diagnosis of homocystinuria } \\
\text { due to MTHFR deficiency } \\
\text { achieved. } \\
\text { Genetic testing of sibling } \\
\text { undertaken and found to be } \\
\text { affected. }\end{array}$ & $\begin{array}{l}\text { ^Skin biopsy, fibroblast culture, enzyme } \\
\text { assay in fibroblasts and single gene } \\
\text { mutation analysis }\end{array}$ & None \\
\hline 25 & $1-11$ years & $\begin{array}{l}\text { Long standing developmental delay and } \\
\text { encephalopathy }\end{array}$ & $\begin{array}{l}\text { MTHFR c.1530G>A } \\
{[\text { p.(Lys510Lys) }]^{* *}}\end{array}$ & hom & $\begin{array}{l}\text { Diagnosis of homocystinuria } \\
\text { due to MTHFR deficiency } \\
\text { achieved }\end{array}$ & $\begin{array}{l}\text { ^Skin biopsy, fibroblast culture, enzyme } \\
\text { assay in fibroblasts and single gene } \\
\text { mutation analysis }\end{array}$ & None \\
\hline 26 & $<1$ year & $\begin{array}{l}\text { Lactic acidosis with methylmalonic aciduria and } \\
\text { elevated plasma homocysteine. Poor feeding } \\
\text { and lethargy. Suspected cbIC disease. }\end{array}$ & $\begin{array}{l}\text { MMACHC c.271dupA } \\
\text { [p.(Arg9|LysfsTerl4)] }\end{array}$ & hom & $\begin{array}{l}\text { Diagnosis of cblC type } \\
\text { methylmalonic aciduria and } \\
\text { homocystinuria, confirmed }\end{array}$ & $\begin{array}{l}\text { ^Skin biopsy, fibroblast culture, } \\
\text { complementation assays and sequencing of } \\
\text { specific gene, or sequential mutation } \\
\text { analysis of multiple genes }\end{array}$ & None \\
\hline \multicolumn{8}{|c|}{ Single sub-panel tested: (AA, NT) } \\
\hline 27 & $1-11$ years & $\begin{array}{l}\text { Macrocephaly, elevated cystine and lysine in } \\
\text { urine. Suspected cystinuria or lysinuric protein } \\
\text { intolerance. }\end{array}$ & $\begin{array}{l}\text { SLC7A9 c.614dupA } \\
\text { [p.(Asn206GlufsTer3)] }\end{array}$ & het & $\begin{array}{l}\text { Carrier finding or possible } \\
\text { diagnosis of cystinuria (but no } \\
\text { second variant found) }\end{array}$ & Single gene mutation analysis & None \\
\hline 28 & $<1$ year & $\begin{array}{l}\text { Hypotonia and poor feeding on day } 1 \text { of life, } \\
\text { hiccups, apnoeas, absent corpus callosum, burst } \\
\text { suppression EEG. Elevated CSF glycine. } \\
\text { Suspected non-ketotic hyperglycinaemia. }\end{array}$ & $\begin{array}{l}\text { GLDC c.1545G>C } \\
\text { [p.(Arg515Ser)], whole gene } \\
\text { deletion }\end{array}$ & $\begin{array}{l}\text { hemizygo } \\
\text { us }\end{array}$ & $\begin{array}{l}\text { Diagnosis of non-ketotic } \\
\text { hyperglycinaemia due to GLDC } \\
\text { deficiency achieved. }\end{array}$ & $\begin{array}{l}\text { ^Liver biopsy, measurement of specific } \\
\text { enzyme activity and/or sequential mutation } \\
\text { analysis of multiple genes }\end{array}$ & None \\
\hline 29 & $<1$ year & $\begin{array}{l}\text { Seizures. Elevated CSF:plasma glycine ratio. } \\
\text { Suspected non-ketotic hyperglycinaemia. }\end{array}$ & $\begin{array}{l}\text { GLDC c.1629delT } \\
\text { [p.(Asn543LysfsTer10)] }\end{array}$ & hom & $\begin{array}{l}\text { Diagnosis of non-ketotic } \\
\text { hyperglycinaemia due to GLDC } \\
\text { deficiency achieved. }\end{array}$ & $\begin{array}{l}\text { ^Liver biopsy, measurement of specific } \\
\text { enzyme activity and/or sequential mutation } \\
\text { analysis of multiple genes }\end{array}$ & None \\
\hline
\end{tabular}




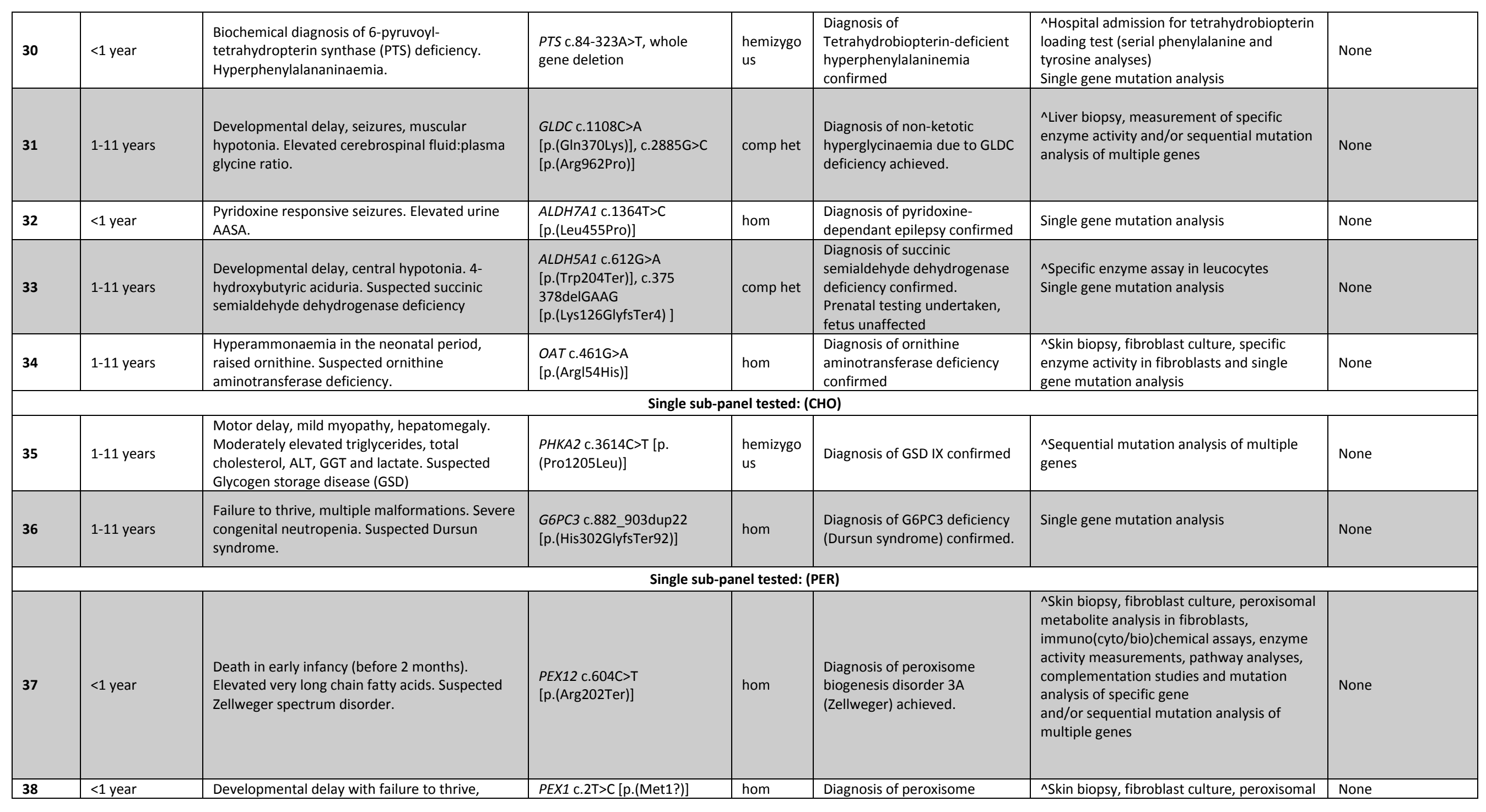




\begin{tabular}{|c|c|c|c|c|c|c|c|}
\hline & & $\begin{array}{l}\text { developmental delay, gastro-oesophageal reflux. } \\
\text { Suspected glycogen synthase deficiency. }\end{array}$ & $\begin{array}{l}\text { [p.(Glu518Ala)], c.729C>A } \\
\text { [p.(Cys243Ter)] }\end{array}$ & & disease 0 confirmed & $\begin{array}{l}\text { and lactate measurements, single gene } \\
\text { mutation analysis }\end{array}$ & \\
\hline \multicolumn{8}{|c|}{ Combination of sub-panels tested: (OA, VIT) (AA,NT) } \\
\hline 44 & $12-17$ years & $\begin{array}{l}\text { Failure to thrive, hyporeflexia, abnormal nerve } \\
\text { conduction. Vitamin B12 responsive } \\
\text { megaloblastic anaemia, methylmalonic aciduria. } \\
\text { Suspected transcobalamin II deficiency. }\end{array}$ & $\begin{array}{l}\text { TCN2 c.497_498delTC } \\
\text { [p.(Leu166ProfsTer7)] }\end{array}$ & hom & $\begin{array}{l}\text { Diagnosis of Transcobalamin II } \\
\text { deficiency confirmed }\end{array}$ & $\begin{array}{l}{ }^{\wedge} \text { Single gene mutation analysis and/or skin } \\
\text { biopsy, fibroblast culture and studies of } \\
\text { transcobalamin synthesis in fibroblasts }\end{array}$ & None \\
\hline \multicolumn{8}{|c|}{ Combination of sub-panels tested: (OA, VIT) (AA, NT) (AMN, FAOD, KET) } \\
\hline 45 & $1-11$ years & $\begin{array}{l}\text { Renal failure, cardiomyopathy. Elevated } \\
\text { methylmalonic acid in urine, abnormal } \\
\text { acylcarnitine profile. Suspected methylmalonic } \\
\text { acidaemia. }\end{array}$ & $\begin{array}{l}\text { MMAA c. } 433 C>T \\
{[p .(A r g 145 T e r)], c .970-2 A>C}\end{array}$ & comp het & $\begin{array}{l}\text { Diagnosis of cblA type } \\
\text { methylmalonic aciduria } \\
\text { achieved }\end{array}$ & $\begin{array}{l}\text { ^Skin biopsy, fibroblast culture, } \\
\text { complementation assays and sequencing of } \\
\text { specific gene, or sequential mutation } \\
\text { analysis of multiple genes }\end{array}$ & None \\
\hline \multicolumn{8}{|c|}{ Combination of sub-panels tested: (OA, VIT) (AMN, FAOD, KET) } \\
\hline 46 & $1-11$ years & $\begin{array}{l}\text { Hypoketotic hypoglycaemia, } \\
\text { hyperammonaemia, supraventricular } \\
\text { tachycardia and Reye-like illness. Suspected } \\
\text { carnitine palmitoyltransferase IA (CPT1A) } \\
\text { deficiency. }\end{array}$ & $\begin{array}{l}\text { CPT1A c.733C >T } \\
{[\text { p.(Arg245Ter)] }}\end{array}$ & hom & Diagnosis of CPT $1 \mathrm{~A}$ confirmed & Single gene mutation analysis & None \\
\hline \multicolumn{8}{|c|}{ All sub-panels tested } \\
\hline 47 & $\begin{array}{l}\text { Adult }(18+ \\
\text { years) }\end{array}$ & $\begin{array}{l}\text { Generalised epilepsy and learning difficulties. } \\
\text { Hydrocephalus diagnosed at } 6 \text { weeks. Mildly } \\
\text { coarse facies, short stature. White cell enzymes } \\
\text { normal. }\end{array}$ & $\begin{array}{l}\text { FAH c. } 648 \mathrm{C}>\mathrm{G} \\
\text { [p.(lle216Met)] }\end{array}$ & hom & $\begin{array}{l}\text { Possible incidental diagnosis of } \\
\text { Tyrosinaemia type I (no } \\
\text { biochemical evidence) }\end{array}$ & None & None \\
\hline 48 & $<1$ year & $\begin{array}{l}\text { Gastroschisis. Investigated for conjugated } \\
\text { hyperbilirubinaemia, urine organic acids } \\
\text { revealed methylmalonic aciduria and raised } \\
\text { malonic acid levels. Suspected methylmalonic } \\
\text { acidaemia. }\end{array}$ & $\begin{array}{l}\text { ACSF3 c.782G>A } \\
\text { [p.(Gly261Glu)], c.1672C>T } \\
\text { [p.(Arg558Trp)] }\end{array}$ & comp het & $\begin{array}{l}\text { Diagnosis of Combined malonic } \\
\text { and methylmalonic aciduria } \\
\text { achieved }\end{array}$ & $\begin{array}{l}\text { ^Skin biopsy, fibroblast culture, } \\
\text { complementation assays and sequencing of } \\
\text { specific gene, or sequential mutation } \\
\text { analysis of multiple genes }\end{array}$ & None \\
\hline 49 & $<1$ year & $\begin{array}{l}\text { Jaundice, hepatosplenomegaly, growth failure, } \\
\text { features of haemophagocytic } \\
\text { lymphohistiocytosis. Elevated ALT, } \\
\text { coagulopathy, reduced leukocyte LAL activity. }\end{array}$ & $\begin{array}{l}\text { LIPA c.824C>T } \\
\text { [p.(Ser275Phe)] }\end{array}$ & hom & $\begin{array}{l}\text { Diagnosis of Wolman disease } \\
\text { confirmed. } \\
\text { Prenatal testing undertaken, } \\
\text { fetus unaffected } \\
\end{array}$ & Single gene mutation analysis & None \\
\hline 50 & $1-11$ years & $\begin{array}{l}\text { Global developmental delay, cerebral atrophy, } \\
\text { thin corpus callosum, hypertonia, delayed visual } \\
\text { maturity. Low CSF 5-methyltetrahydrofolate. } \\
\text { Suspected cerebral folate deficiency }\end{array}$ & FOLR1 c. $493+2 \mathrm{~T}>\mathrm{C}$ & het & $\begin{array}{l}\text { Possible diagnosis of cerebral } \\
\text { folate transport deficiency (no } \\
\text { second variant found) }\end{array}$ & Single gene mutation analysis & None \\
\hline 51 & $1-11$ years & $\begin{array}{l}\text { Global developmental delay, bilateral retinal } \\
\text { dystrophy, unilateral cataract, umbilical hernia, } \\
\text { hepatosplenomegaly, hypothyroidism, } \\
\text { prolonged hyperbilirubinaemia. }\end{array}$ & $\begin{array}{l}\text { ACOX1 c.443G }>A \\
{[p .(\operatorname{Arg} 148 G \ln )]} \\
\text { GALK1 c.479C >T }\end{array}$ & hom & $\begin{array}{l}\text { Possible diagnosis of } \\
\text { peroxisomal acyl-CoA oxidase } \\
\text { deficiency }\end{array}$ & None & $\begin{array}{l}\text { Further } \\
\text { confirmatory } \\
\text { biochemical testing } \\
\text { could not be } \\
\text { performed as }\end{array}$ \\
\hline
\end{tabular}


[p.(Ser160Leu)]

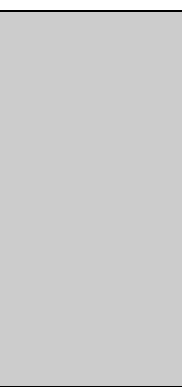

Possible diagnosis of

galactokinase deficiency with

cataracts patient lost to

follow up. May have

required skin

biopsy, fibroblast

culture, peroxisomal

specific enzyme

activity, galactose

and galactitol in

blood and urine,

galactokinase

activity in blood. 


\section{Acknowledgements}

This work was supported by the Manchester Biomedical Research Centre, the British Inherited Metabolic Disease Group 2015 Studentship scheme and the Central Manchester NHS Foundation Trust Newly Appointed Consultants Leadership Programme 2014. We thank members of the Willink Biochemical laboratory, Ms Teresa Wu, Ms Karen Tylee, Dr Heather Church and Dr Mick Henderson for help in providing the biochemical data. We thank Juan Pié, University of Zaragoza, Spain for providing HMG-CoA synthase enzyme activity data. We acknowledge Ms Georgina Hall and Ms Nasaim Khan for their contribution to the multi-disciplinary team meetings. We express our gratitude to Dr Ashique Ahamed, Dr Ben Grey, Dr Simon Hellings, Dr Lamiya Mohiyiddeen and Ms Liz Oldfield-Beechey for useful discussions on the project. 


\section{REFERENCES}

1. Applegarth DA, Toone JR, Lowry RB. Incidence of inborn errors of metabolism in British Columbia, 1969-1996. Pediatrics 2000;105(1):e10

2. Sanderson S, Green A, Preece MA, et al. The incidence of inherited metabolic disorders in the West Midlands, UK. Arch Dis Child 2006;91(11):896-9 doi: 10.1136/adc.2005.091637[published Online First: Epub Date]|.

3. McDonald L, Rennie A, Tolmie J, et al. Investigation of global developmental delay. Archives of Disease in Childhood 2006;91(8):701-05 doi: 10.1136/adc.2005.078147[published Online First: Epub Date]|.

4. van Karnebeek CD, Shevell M, Zschocke J, et al. The metabolic evaluation of the child with an intellectual developmental disorder: diagnostic algorithm for identification of treatable causes and new digital resource. Mol Genet Metab 2014;111(4):428-38 doi: 10.1016/j.ymgme.2014.01.011[published Online First: Epub Date]|.

5. van Karnebeek CD, Houben RF, Lafek M, et al. The treatable intellectual disability APP http://www.treatable-id.org/ a digital tool to enhance diagnosis \& care for rare diseases. Orphanet J Rare Dis 2012;7:47 doi: 10.1186/1750-1172-747[published Online First: Epub Date]|.

6. Banka S, Blom HJ, Walter J, et al. Identification and characterization of an inborn error of metabolism caused by dihydrofolate reductase deficiency. American journal of human genetics 2011;88(2):216-25 doi:

10.1016/j.ajhg.2011.01.004[published Online First: Epub Date]|.

7. Banka S, de Goede C, Yue WW, et al. Expanding the clinical and molecular spectrum of thiamine pyrophosphokinase deficiency: a treatable neurological disorder caused by TPK1 mutations. Mol Genet Metab 2014;113(4):301-6 doi: 10.1016/j.ymgme.2014.09.010[published Online First: Epub Date]|.

8. Banka S, Newman WG. A clinical and molecular review of ubiquitous glucose-6phosphatase deficiency caused by G6PC3 mutations. Orphanet J Rare Dis 2013;8:84 doi: 10.1186/1750-1172-8-84[published Online First: Epub Date]|.

9. van Karnebeek CD, Stockler S. Treatable inborn errors of metabolism causing intellectual disability: a systematic literature review. Mol Genet Metab 2012;105(3):368-81 doi: 10.1016/j.ymgme.2011.11.191[published Online First: Epub Date]|.

10. Cleary MA, Green A. Developmental delay: when to suspect and how to investigate for an inborn error of metabolism. Arch Dis Child 2005;90(11):1128-32 doi: 10.1136/adc.2005.072025[published Online First: Epub Date]|.

11. Argmann CA, Houten SM, Zhu J, et al. A Next Generation Multiscale View of Inborn Errors of Metabolism. Cell metabolism 2016;23(1):13-26 doi: 10.1016/j.cmet.2015.11.012[published Online First: Epub Date]|.

12. Yang Y, Muzny DM, Reid JG, et al. Clinical Whole-Exome Sequencing for the Diagnosis of Mendelian Disorders. The New England journal of medicine 2013;369(16):1502-11 doi: 10.1056/NEJMoa1306555[published Online First: Epub Date]|.

13. Vrijenhoek T, Kraaijeveld K, Elferink M, et al. Next-generation sequencing-based genome diagnostics across clinical genetics centers: implementation choices and their effects. European Journal of Human Genetics 2015;23(9):1142-50 doi: 10.1038/ejhg.2014.279[published Online First: Epub Date]|. 
14. Berg JS, Evans JP, Leigh MW, et al. Next generation massively parallel sequencing of targeted exomes to identify genetic mutations in primary ciliary dyskinesia: implications for application to clinical testing. Genetics in medicine : official journal of the American College of Medical Genetics 2011;13(3):218-29 doi: 10.1097/GIM.0b013e318203cff2[published Online First: Epub Date]|.

15. Jones MA, Bhide S, Chin E, et al. Targeted PCR-based enrichment and next generation sequencing for diagnostic testing of congenital disorders of glycosylation (CDG). Genetics in Medicine 2011;13(11):921-32 doi: 10.1097/GIM.0b013e318226fbf2[published Online First: Epub Date]|.

16. Meder B, Haas J, Keller A, et al. Targeted next-generation sequencing for the molecular genetic diagnostics of cardiomyopathies. Circulation Cardiovascular genetics 2011;4(2):110-22 doi: 10.1161/circgenetics.110.958322[published Online First: Epub Date]|.

17. Simpson DA, Clark GR, Alexander S, et al. Molecular diagnosis for heterogeneous genetic diseases with targeted high-throughput DNA sequencing applied to retinitis pigmentosa. Journal of medical genetics 2011;48(3):145-51 doi: 10.1136/jmg.2010.083568[published Online First: Epub Date]|.

18. Matthijs $G$, Souche $E$, Alders $M$, et al. Guidelines for diagnostic next-generation sequencing. European journal of human genetics : EJHG 2016;24(1):2-5 doi: 10.1038/ejhg.2015.226[published Online First: Epub Date]|.

19. Saudubray J-M, van den Berghe G, Walter JH, editors. Inborn Metabolic Diseases. 5th ed: Springer-Verlag Berlin Heidelberg, 2012.

20. Ellingford JM, Barton S, Bhaskar S, et al. Molecular findings from 537 individuals with inherited retinal disease. Journal of medical genetics 2016 doi: 10.1136/jmedgenet-2016-103837[published Online First: Epub Date]|.

21. Plagnol V, Curtis J, Epstein $M$, et al. A robust model for read count data in exome sequencing experiments and implications for copy number variant calling. Bioinformatics 2012;28(21):2747-54 doi: 10.1093/bioinformatics/bts526[published Online First: Epub Date]|.

22. Jones $M A$, Rhodenizer $D$, da Silva $C$, et al. Molecular diagnostic testing for congenital disorders of glycosylation (CDG): detection rate for single gene testing and next generation sequencing panel testing. Mol Genet Metab 2013;110(1-2):78-85 doi: 10.1016/j.ymgme.2013.05.012[published Online First: Epub Date]|.

23. Sun Y, Ruivenkamp CA, Hoffer MJ, et al. Next-generation diagnostics: gene panel, exome, or whole genome? Human mutation 2015;36(6):648-55 doi: 10.1002/humu.22783[published Online First: Epub Date]|.

24. Xue $Y$, Ankala A, Wilcox WR, et al. Solving the molecular diagnostic testing conundrum for Mendelian disorders in the era of next-generation sequencing: single-gene, gene panel, or exome/genome sequencing. Genetics in medicine : official journal of the American College of Medical Genetics 2015;17(6):444-51 doi: 10.1038/gim.2014.122[published Online First: Epub Date]|.

25. Ferdinandusse S, Ebberink MS, Vaz FM, et al. The important role of biochemical and functional studies in the diagnostics of peroxisomal disorders. J Inherit Metab Dis 2016 doi: 10.1007/s10545-016-9922-4[published Online First: Epub Date]|. 
26. Fowler B, Leonard JV, Baumgartner MR. Causes of and diagnostic approach to methylmalonic acidurias. J Inherit Metab Dis 2008;31(3):350-60 doi: 10.1007/s10545-008-0839-4[published Online First: Epub Date]|.

27. Morris AA, Thekekara A, Wilks Z, et al. Evaluation of fasts for investigating hypoglycaemia or suspected metabolic disease. Arch Dis Child 1996;75(2):115-9

28. Chow SL, Gandhi V, Krywawych S, et al. The significance of a high plasma ammonia value. Archives of Disease in Childhood 2004;89(6):585-86 doi: 10.1136/adc.2003.036236[published Online First: Epub Date]|.

29. Ghosh A, Banerjee I, Morris AA. Recognition, assessment and management of hypoglycaemia in childhood. Arch Dis Child 2016;101(6):575-80 doi: 10.1136/archdischild-2015-308337[published Online First: Epub Date]|.

30. de Goede C, Yue WW, Yan G, et al. Role of reverse phenotyping in interpretation of next generation sequencing data and a review of INPP5E related disorders. European journal of paediatric neurology : EJPN : official journal of the European Paediatric Neurology Society 2016;20(2):286-95 doi: 10.1016/j.ejpn.2015.11.012[published Online First: Epub Date]|.

31. Wortmann SB, Koolen DA, Smeitink JA, et al. Whole exome sequencing of suspected mitochondrial patients in clinical practice. J Inherit Metab Dis 2015;38(3):437-43 doi: 10.1007/s10545-015-9823-y[published Online First: Epub Date]|.

32. Legati A, Reyes A, Nasca A, et al. New genes and pathomechanisms in mitochondrial disorders unraveled by NGS technologies. Biochimica et biophysica acta 2016 doi: 10.1016/j.bbabio.2016.02.022[published Online First: Epub Date]|.

33. Stranneheim H, Engvall M, Naess K, et al. Rapid pulsed whole genome sequencing for comprehensive acute diagnostics of inborn errors of metabolism. BMC genomics 2014;15:1090 doi: 10.1186/1471-2164-151090[published Online First: Epub Date]|.

34. Pupavac M, Tian X, Chu J, et al. Added value of next generation gene panel analysis for patients with elevated methylmalonic acid and no clinical diagnosis following functional studies of vitamin B12 metabolism. Mol Genet Metab 2016;117(3):363-8 doi: 10.1016/j.ymgme.2016.01.008[published Online First: Epub Date]|.

35. Stitziel NO, Peloso GM, Abifadel M, et al. Exome sequencing in suspected monogenic dyslipidemias. Circulation Cardiovascular genetics 2015;8(2):34350 doi: 10.1161/circgenetics.114.000776[published Online First: Epub Date]|.

36. Lieber DS, Calvo SE, Shanahan K, et al. Targeted exome sequencing of suspected mitochondrial disorders. Neurology 2013;80(19):1762-70 doi: 10.1212/WNL.0b013e3182918c40[published Online First: Epub Date]|.

37. Tarailo-Graovac M, Shyr C, Ross $\mathrm{CJ}$, et al. Exome Sequencing and the Management of Neurometabolic Disorders. New England Journal of Medicine 2016;374(23):2246-55 doi: 10.1056/NEJMoa1515792[published Online First: Epub Date]|.

38. Taylor RW, Pyle A, Griffin $\mathrm{H}$, et al. Use of whole-exome sequencing to determine the genetic basis of multiple mitochondrial respiratory chain complex deficiencies. Jama 2014;312(1):68-77 doi: 10.1001/jama.2014.7184[published Online First: Epub Date]|. 
39. Yubero D, Brandi N, Ormazabal A, et al. Targeted Next Generation Sequencing in Patients with Inborn Errors of Metabolism. PloS one 2016;11(5):e0156359 doi: 10.1371/journal.pone.0156359[published Online First: Epub Date]|.

40. Cosma MP, Pepe S, Parenti G, et al. Molecular and functional analysis of SUMF1 mutations in multiple sulfatase deficiency. Human mutation 2004;23(6):57681 doi: 10.1002/humu.20040[published Online First: Epub Date]|.

41. Burda P, Schafer A, Suormala T, et al. Insights into severe $5,10-$ methylenetetrahydrofolate reductase deficiency: molecular genetic and enzymatic characterization of 76 patients. Human mutation 2015;36(6):61121 doi: 10.1002/humu.22779[published Online First: Epub Date]|. 


\section{FIGURE LEGENDS}

Figure 1: Frequency of diagnosis by sub-panels and biochemical markers:

(A) Proportion of individuals in whom primary pathogenic variants and incidental carrier findings were identified according to whether specific gene defects or subgroups of IEMs were suspected and sub-panels requested. 'Specific sub-group of IEM suspected, single sub-panel tested' refers to individuals in whom the differential diagnosis included more than one gene but only a single sub-panel was requested. 'Combination sub-panels' refers to any combination of sub-panels other than all six sub-panels. The frequency of a confirmed genetic diagnosis was significantly higher when a single specific gene defect or a specific sub-group of IEM was suspected, in comparison with suspected IEM tested by requests for combination or all sub-panels $(p<0.0001)$, underlining the complementary role of metabolic and NGS investigations in clinical diagnostics.

(B) Proportion of individuals with primary pathogenic variants according to abnormal biochemical markers detected in screening investigations. The diagnostic yield was much higher for metabolic markers such as MMA, abnormal VLCFA and specific enzyme deficiencies. It was lower for hypoglycaemia and hyperammonaemia, especially if no additional marker was identified in the previous biochemical testing. This suggests that many of these individuals perhaps do not have a Mendelian genetic disorder or many genes influencing glucose and ammonia levels in children are yet to be discovered. 'Any' refers to any biochemical marker(s) that were suggestive of a specific diagnosis or group of disorders. 'Only hypoglycaemia' and 'only hyperammonaemia' refer to the absence of additional markers such as abnormal plasma amino acids, acylcarnitine profile or urine organic acids that suggested a specific diagnosis or group of disorders.

'Heterozygous primary pathogenic variant' refers to individuals in whom a mutation was identified in a heterozygous state in a gene relevant to the reported phenotype, which may reflect a carrier finding or a partial diagnosis.

Key - VLCFA very long chain fatty acids, def deficiency, MMA methylmalonic aciduria

Figure 2: (A) 'Traditional' approach to diagnosis of IEMs. (B) Incorporation of NGS techniques into diagnostic algorithm of IEMs.

Key - WES whole exome sequencing, WGS whole genome sequencing 


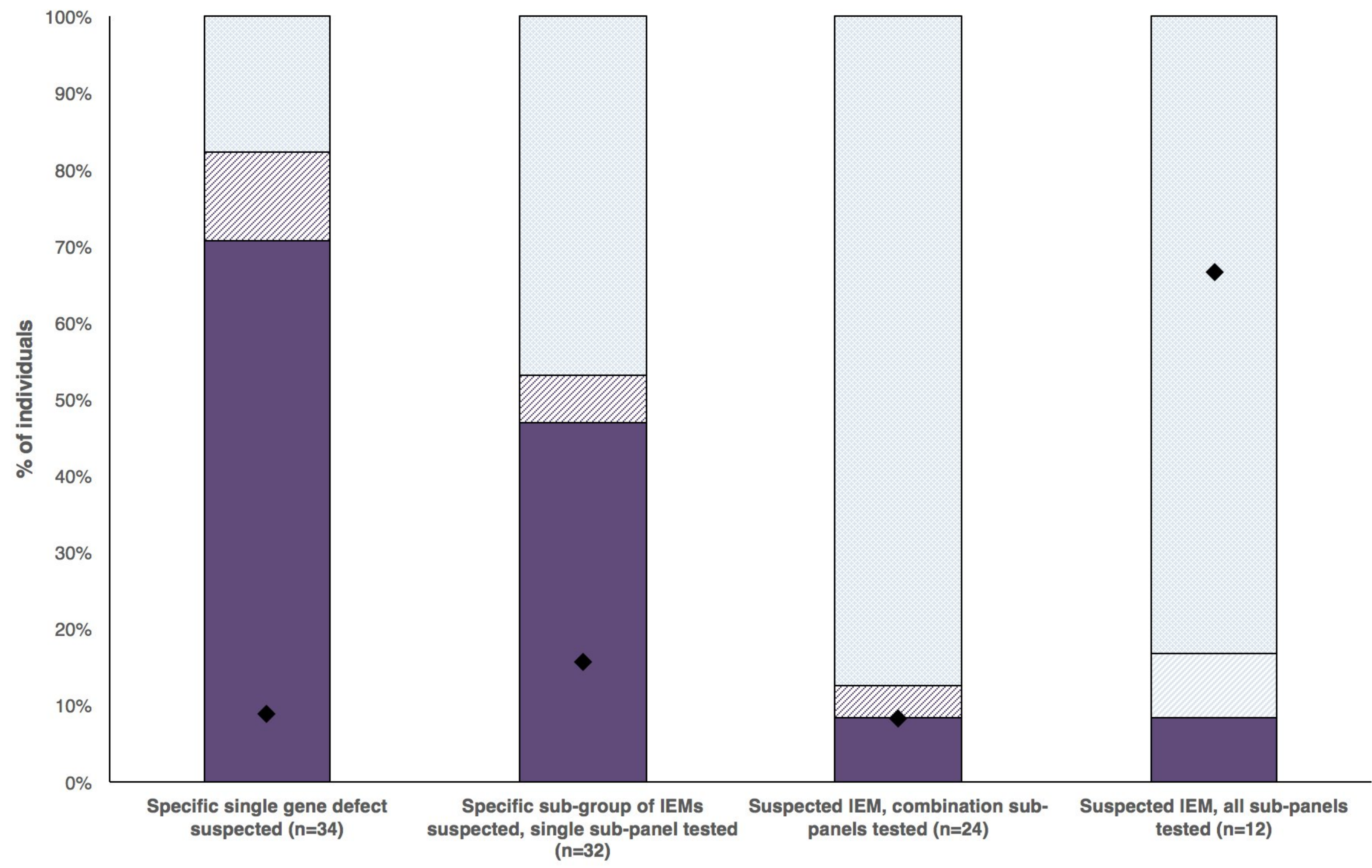

$\square$ Complete diagnosis $\square$ Heterozygous primary pathogenic variant $\square$ Diagnosis uncertain (unclear if fits phenotype) $\square$ No diagnosis $\bullet$ Incidental carrier finding 


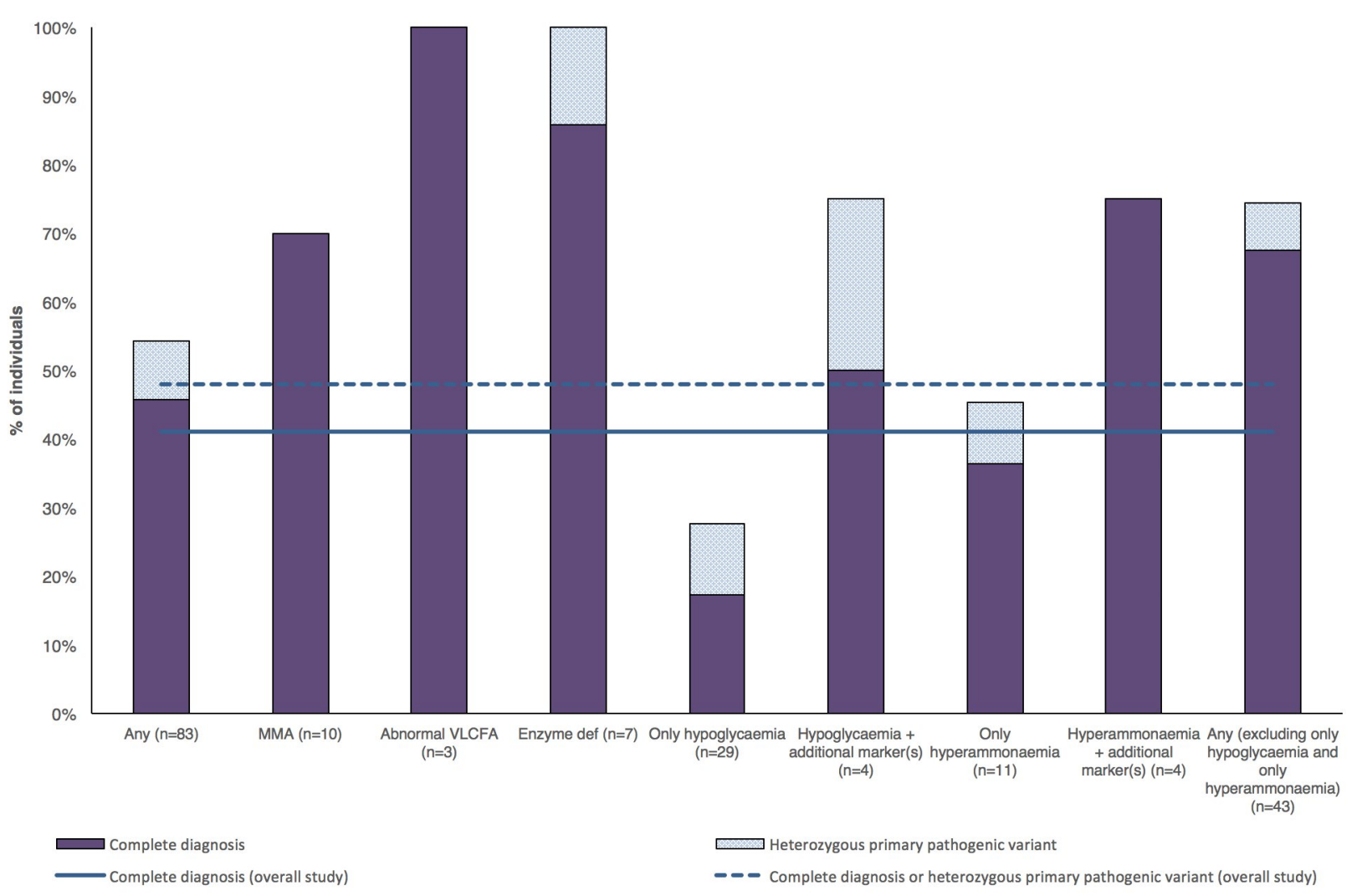




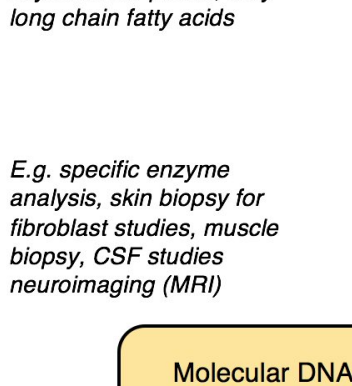
long chain fatty acids

E.g. specific enzyme analysis, skin biopsy for fibroblast studies, muscle biopsy, CSF studies neuroimaging (MRI)

E.g. ammonia, lactate, urine organic acids, plasma amino acids, acylcarnitine profile, very 


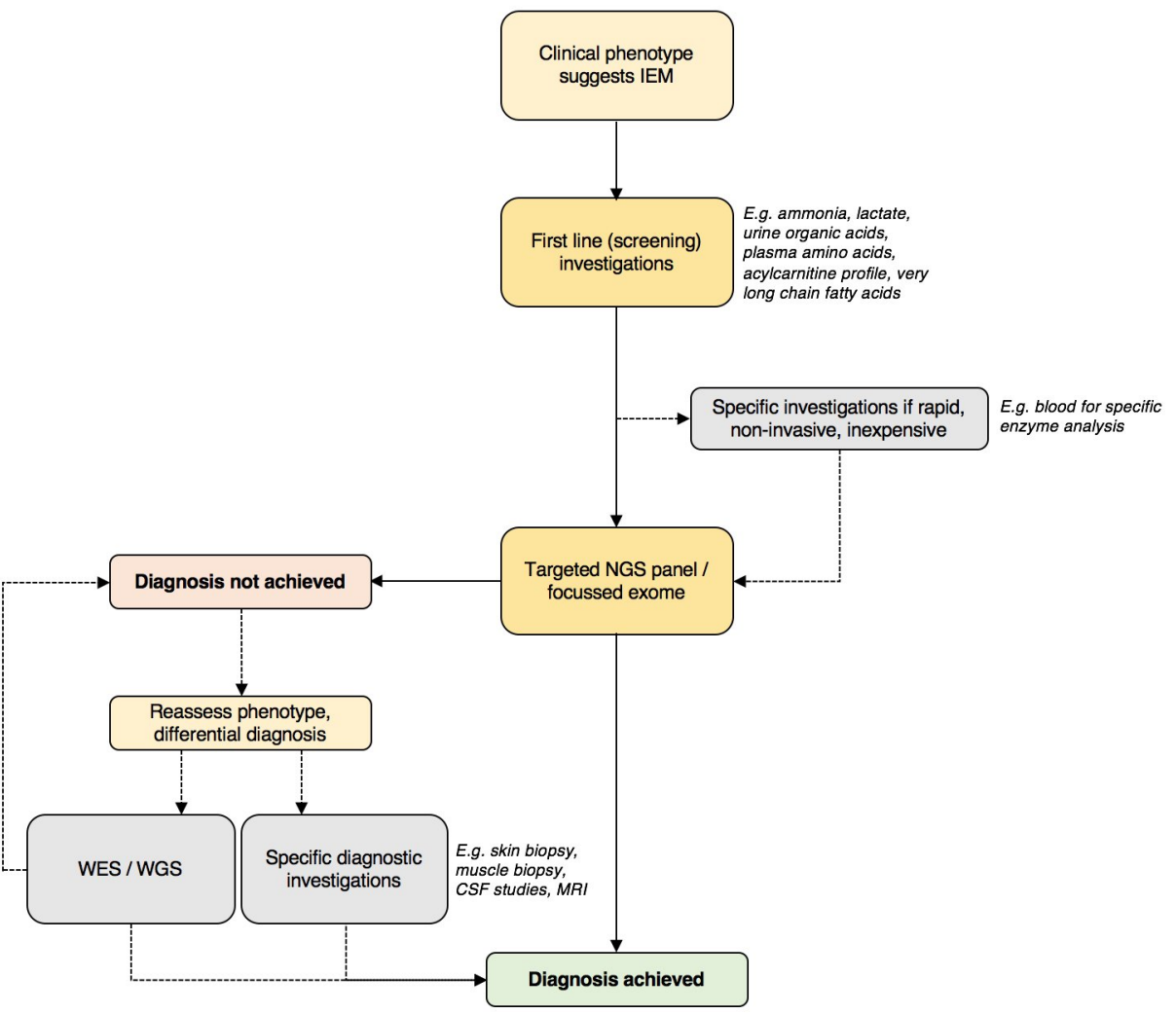


Diagnosing Childhood-onset Inborn Errors of Metabolism by Next Generation Sequencing 


\section{CLINICAL PROFORMA FOR MANCHESTER METABOLIC NGS PANELS}

Patient Name:

Sex: Male $\square$ Female $\square \quad$ Date of Birth (D/M/Y): $\quad$ Reference Number:

\begin{tabular}{|l|l|}
\hline Clinical Information & \\
\hline Clinical features & \\
\hline Age of onset & \\
\hline $\begin{array}{l}\text { Details of relevant biochemical } \\
\text { testing }\end{array}$ & Dominant $\square \quad$ X-linked $\square \quad$ Recessive $\square \quad$ Sporadic $\square \quad$ Information not available $\square$ \\
\hline \begin{tabular}{l} 
Likely mode of inheritance \\
\hline $\begin{array}{l}\text { Relevant family history } \\
\text { (draw brief pedigrees if needed) } \\
\text { and any other relevant } \\
\text { information }\end{array}$
\end{tabular} \\
\hline $\begin{array}{l}\text { Possible or likely diagnosis or } \\
\text { disease group }\end{array}$ \\
\hline $\begin{array}{l}\text { Is the patient known to any } \\
\text { Consultants in Manchester } \\
\text { Genetics department? } \\
\text { (If yes, give name) }\end{array}$ \\
\hline
\end{tabular}

\begin{tabular}{|c|c|c|}
\hline \multicolumn{3}{|l|}{ Gene panel request } \\
\hline $\begin{array}{l}\text { AA + NT } \\
\text { AMN + FAOD + KET } \\
O A+V I T \\
\text { CHO } \\
\text { LSD + NCL } \\
\text { PER } \\
\text { Full Panel }\end{array}$ & $\begin{array}{l}\square \\
\square \\
\square \\
\square \\
\square \\
\square \\
\square\end{array}$ & $\begin{array}{l}\text { Key: AA Disorders of amino acid metabolism and cerebral organic acid } \\
\text { disorders; NT Disorders of neurotransmission; AMN Disorders associated } \\
\text { with hyperammonaemia; FAOD Fatty acid oxidation defects; KET Disorders } \\
\text { of ketogenesis or ketolysis; OA Organic acidaemias, including disorders of } \\
\text { branched chain amino acid catabolism, 3-methylglutaconic acidurias; VIT } \\
\text { Folate and cobalamin defects, also riboflavin transport defects, and biotin- } \\
\text { responsive disorders; CHO Disorders of carbohydrate metabolism; LSD } \\
\text { Lysosomal disorders; NCL Neuronal ceroid lipofuscinoses; PER Peroxisomal } \\
\text { biogenesis disorders and disorders of single peroxisomal enzymes } \\
\text { For full list of genes in each panel please see www.mangen.co.uk }\end{array}$ \\
\hline \multirow[t]{2}{*}{ Signature } & & Department \\
\hline & & Hospital \\
\hline Name & & Phone \\
\hline Date & & Email \\
\hline
\end{tabular}


MANCHESTER

www.mangen.org.uk

\section{Central Manchester University Hospitals WHS}

NHS Foundation Trust

\section{Manchester Centre for Genomic Medicine \\ Clinical Genetics Service \\ $6^{\text {th }}$ Floor, Saint Mary's Hospital \\ Oxford Road \\ Manchester \\ M13 9WL}

Tel: 01612766506 Fax: 01612766145

\section{Manchester Metabolic NGS Panel Test Consent Form}

I, (DOB

agree for myself/ (DOB

to undergo genetic testing for the Manchester Metabolic NGS Panel.

I understand:

- The genetic test looks at many different metabolic genes.

- The test may or may not find the cause of the metabolic disorder.

- The test may find changes in other genes that are not causing the metabolic disorder.

- The test may have implications for my wider family.

- The test may reveal a change in a gene that has other health implications in addition to the metabolic disorder.

- The test may reveal a change that cannot be interpreted. In these cases, family studies may be helpful.

- The genetic doctor / counsellor will explain the results of the test and its implications for me and my family as far as current knowledge allows.

I agree to my sample being stored in case future relevant tests become available and that my test result may be used by health care professionals to help other family members.

I agree for my GP and other health professionals to be informed of the test result.

I have been given an information sheet entitled 'Manchester Metabolic NGS panel testing: Information for patients'

Date

Relationship with the patient - Patient/Father/Mother/Guardian/Other (please specify

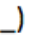

Clinician signature Date 
Supplementary Table S1: Genes reported since 2013 to be implicated in mitochondrial disorders or congenital disorders of glycosylation demonstrating that the genetic heterogeneity of these group of disorders remains to be resolved.

\begin{tabular}{|c|c|c|}
\hline Gene & Disorder & Reference \\
\hline$C O A-X$ & Mitochondrial & Legati (2016) \\
\hline CYP2U1 & Mitochondrial & Legati (2016) \\
\hline E4F1 & Mitochondrial & Legati (2016) \\
\hline$C L P B$ & Mitochondrial & Wortmann (2015a) \\
\hline PARS2 & Mitochondrial & Sohou (2015) \\
\hline NARS2 & Mitochondrial & Sohou (2015) \\
\hline$F B X L 4$ & Mitochondrial & Gai (2013) \\
\hline TMEM126B & Mitochondrial & Sanchez-Cabellero (2016) \\
\hline NAXE & Mitochondrial & Spiegel (2016) \\
\hline NDUFB11 & Mitochondrial & van Rahden (2015) \\
\hline LYRM7 & Mitochondrial & Invernizzi (2013) \\
\hline TTC19 & Mitochondrial & Atwal (2014) \\
\hline GYG2 & Mitochondrial & Imagawa (2014) \\
\hline PET100 & Mitochondrial & $\operatorname{Lim}(2014)$ \\
\hline NUDFA4 & Mitochondrial & Pitceathly (2013) \\
\hline VARS2 & Mitochondrial & Taylor (2014) \\
\hline GARS & Mitochondrial & Taylor (2014) \\
\hline FLAD1 & Mitochondrial & Taylor (2014) \\
\hline PTCD1 & Mitochondrial & Taylor (2014) \\
\hline SLC25A42 & Mitochondrial & Shamseldin (2016) \\
\hline CHKB & Mitochondrial & Castro-Gago (2014) \\
\hline$C A D$ & Congenital disorder of glycosylation & $\mathrm{Ng}(2015)$ \\
\hline STT3A & Congenital disorder of glycosylation & Shrimal (2013) \\
\hline STT3B & Congenital disorder of glycosylation & Shrimal (2013) \\
\hline NUS1 & Congenital disorder of glycosylation & Park (2014) \\
\hline SSR4 & Congenital disorder of glycosylation & Losfeld (2014) \\
\hline MAN1B1 & Congenital disorder of glycosylation & van Scherpenzeel (2014) \\
\hline SLC35A1 & Congenital disorder of glycosylation & Mohamed (2013) \\
\hline TMEM165 & Congenital disorder of glycosylation & Zeevaert (2013) \\
\hline SLC35A2 & Congenital disorder of glycosylation & Kodera (2013) \\
\hline PGM3 & Congenital disorder of glycosylation & Zhang (2014) \\
\hline CCDC115 & Congenital disorder of glycosylation & Jansen (2016a) \\
\hline SLC39A8 & Congenital disorder of glycosylation & Boycott (2015) \\
\hline TMEM199 & Congenital disorder of glycosylation & Jansen (2016b) \\
\hline
\end{tabular}


sub-panels.

Key - AA Disorders of amino acid metabolism including phenylketonuria, and cerebral organic acid disorders; AMN Disorders associated with hyperammonaemia; CDS Coding DNA Sequence; CHO Disorders of carbohydrate metabolism; FAOD Fatty acid oxidation defects including multiple acyl-COA dehydrogenase deficiency; KET Disorders of ketogenesis or ketolysis; LSD Lysosomal disorders: includes lysosomal storage disorders, transport defects and protease defects; NCL Neuronal ceroid lipofuscinoses; NT Disorders of neurotransmission (includes pterins, tyrosinaemia); OA Organic acidaemias, including disorders of branched chain amino acid catabolism, 3-methylglutaconic acidurias; PER Peroxisomal biogenesis disorders and disorders of single peroxisomal enzymes; ROI Regions of Interest, VIT Folate and cobalamin defects, also riboflavin transport defects, and biotin-responsive disorders; Note that some genes are included in more than one sub-panel.

\begin{tabular}{|c|c|c|c|c|c|c|}
\hline Gene & $\begin{array}{l}\text { CDS } \\
\text { Size }\end{array}$ & $\begin{array}{l}\text { Number of } \\
\text { Exons }\end{array}$ & ROI Size & $\begin{array}{l}\text { Gene OMIM } \\
\#\end{array}$ & Sub-panel & Additional sub-panel \\
\hline$O A T$ & 1320 & 10 & 2320 & 613349 & $\mathrm{AA}, \mathrm{NT}$ & AMN, FAOD, KET \\
\hline SLC7A7 & 1536 & 11 & 2636 & 603593 & $\mathrm{AA}, \mathrm{NT}$ & AMN, FAOD, KET \\
\hline PTS & 438 & 6 & 1038 & 612719 & $\mathrm{AA}, \mathrm{NT}$ & \\
\hline$Q D P R$ & 735 & 7 & 1435 & 612676 & $\mathrm{AA}, \mathrm{NT}$ & \\
\hline GCH1 & 753 & 6 & 1353 & 600225 & $\mathrm{AA}, \mathrm{NT}$ & \\
\hline PCBD1 & 315 & 4 & 715 & 126090 & $\mathrm{AA}, \mathrm{NT}$ & \\
\hline$F A H$ & 1260 & 15 & 2760 & 613871 & $\mathrm{AA}, \mathrm{NT}$ & \\
\hline ALDH5A1 & 1647 & 11 & 2747 & 610045 & $\mathrm{AA}, \mathrm{NT}$ & \\
\hline GCDH & 1317 & 12 & 2517 & 608801 & $\mathrm{AA}, \mathrm{NT}$ & \\
\hline ALDH18A1 & 2388 & 18 & 4188 & 138250 & $\mathrm{AA}, \mathrm{NT}$ & \\
\hline$P A H$ & 1359 & 13 & 2659 & 612349 & $\mathrm{AA}, \mathrm{NT}$ & \\
\hline TAT & 1365 & 12 & 2565 & 613018 & $\mathrm{AA}, \mathrm{NT}$ & \\
\hline$H P D$ & 1182 & 14 & 2582 & 609695 & $\mathrm{AA}, \mathrm{NT}$ & \\
\hline$H G D$ & 1338 & 14 & 2738 & 607474 & $\mathrm{AA}, \mathrm{NT}$ & \\
\hline CBS & 1698 & 18 & 3498 & 613381 & $\mathrm{AA}, \mathrm{NT}$ & \\
\hline MAT1A & 1188 & 9 & 2088 & 610550 & $\mathrm{AA}, \mathrm{NT}$ & \\
\hline GNMT & 888 & 6 & 1488 & 606628 & $\mathrm{AA}, \mathrm{NT}$ & \\
\hline$A H C Y$ & 1299 & 10 & 2299 & 180960 & $\mathrm{AA}, \mathrm{NT}$ & \\
\hline CTH & 1218 & 12 & 2418 & 607657 & $\mathrm{AA}, \mathrm{NT}$ & \\
\hline SUOX & 1638 & 4 & 2038 & 606887 & $\mathrm{AA}, \mathrm{NT}$ & \\
\hline L2HGDH & 1392 & 10 & 2392 & 609584 & $\mathrm{AA}, \mathrm{NT}$ & \\
\hline$D 2 H G D H$ & 1566 & 10 & 2566 & 609186 & $\mathrm{AA}, \mathrm{NT}$ & \\
\hline$A S P A$ & 942 & 7 & 1642 & 608034 & $\mathrm{AA}, \mathrm{NT}$ & \\
\hline NAT8L & 909 & 3 & 1209 & 610647 & $\mathrm{AA}, \mathrm{NT}$ & \\
\hline$A M T$ & 1212 & 9 & 2112 & 238310 & $\mathrm{AA}, \mathrm{NT}$ & \\
\hline GLDC & 3063 & 25 & 5563 & 238300 & $\mathrm{AA}, \mathrm{NT}$ & \\
\hline GCSH & 522 & 5 & 1022 & 238330 & $\mathrm{AA}, \mathrm{NT}$ & \\
\hline SLC3A1 & 2058 & 10 & 3058 & 104614 & $\mathrm{AA}, \mathrm{NT}$ & \\
\hline SLC7A9 & 1464 & 13 & 2764 & 604144 & $\mathrm{AA}, \mathrm{NT}$ & \\
\hline SLC6A19 & 1905 & 12 & 3105 & 608893 & $\mathrm{AA}, \mathrm{NT}$ & \\
\hline SLC36A2 & 1452 & 10 & 2452 & 608331 & $\mathrm{AA}, \mathrm{NT}$ & \\
\hline$A B A T$ & 1548 & 16 & 3148 & 137150 & $\mathrm{AA}, \mathrm{NT}$ & \\
\hline GLRA1 & 1374 & 9 & 2274 & 138491 & $\mathrm{AA}, \mathrm{NT}$ & \\
\hline GABRG2 & 1548 & 11 & 2648 & 137164 & $\mathrm{AA}, \mathrm{NT}$ & \\
\hline SLC25A22 & 972 & 10 & 1972 & 609302 & $\mathrm{AA}, \mathrm{NT}$ & \\
\hline$T H$ & 1587 & 14 & 2987 & 191290 & $\mathrm{AA}, \mathrm{NT}$ & \\
\hline$D D C$ & 1443 & 15 & 2943 & 107930 & $\mathrm{AA}, \mathrm{NT}$ & \\
\hline$D B H$ & 1854 & 12 & 3054 & 609312 & $\mathrm{AA}, \mathrm{NT}$ & \\
\hline MAOA & 1584 & 15 & 3084 & 309850 & $\mathrm{AA}, \mathrm{NT}$ & \\
\hline$S P R$ & 786 & 3 & 1086 & 182125 & $\mathrm{AA}, \mathrm{NT}$ & \\
\hline SLC6A3 & 1863 & 15 & 3363 & 126455 & $\mathrm{AA}, \mathrm{NT}$ & \\
\hline ALDH7A1 & 1620 & 18 & 3420 & 107323 & $\mathrm{AA}, \mathrm{NT}$ & \\
\hline$P N P O$ & 786 & 7 & 1486 & 603287 & $\mathrm{AA}, \mathrm{NT}$ & \\
\hline ETFA & 1002 & 12 & 2202 & 608053 & AMN, FAOD, KET & \\
\hline ETFB & 1041 & 5 & 1541 & 130410 & AMN, FAOD, KET & \\
\hline ETFDH & 1854 & 13 & 3154 & 231675 & AMN, FAOD, KET & \\
\hline NAGS & 1605 & 7 & 2305 & 608300 & AMN, FAOD, KET & \\
\hline CPS1 & 4521 & 39 & 8421 & 608307 & AMN, FAOD, KET & \\
\hline OTC & 1065 & 10 & 2065 & 300461 & AMN, FAOD, KET & \\
\hline ASS1 & 1239 & 15 & 2739 & 603470 & AMN, FAOD, KET & \\
\hline$A S L$ & 1395 & 17 & 3095 & 608310 & AMN, FAOD, KET & \\
\hline ARG1 & 993 & 8 & 1793 & 608313 & AMN, FAOD, KET & \\
\hline SLC25A13 & 2031 & 18 & 3831 & 603859 & AMN, FAOD, KET & \\
\hline SLC25A15 & 906 & 7 & 1606 & 603861 & AMN, FAOD, KET & \\
\hline GLUD1 & 1677 & 13 & 2977 & 606762 & AMN, FAOD, KET & \\
\hline SLC22A5 & 1746 & 11 & 2846 & 603377 & AMN, FAOD, KET & \\
\hline CPT1A & 2322 & 19 & 4222 & 600528 & AMN, FAOD, KET & \\
\hline SLC25A20 & 906 & 9 & 1806 & 613698 & AMN, FAOD, KET & \\
\hline CPT2 & 1977 & 5 & 2477 & 600650 & AMN, FAOD, KET & \\
\hline$A C A D V L$ & 2037 & 21 & 4137 & 609575 & AMN, FAOD, KET & \\
\hline HADHA & 2292 & 20 & 4292 & 600890 & AMN, FAOD, KET & \\
\hline HADHB & 1425 & 16 & 3025 & 143450 & AMN, FAOD, KET & \\
\hline ACADM & 1365 & 13 & 2665 & 607008 & AMN, FAOD, KET & \\
\hline ACADS & 1239 & 10 & 2239 & 606885 & AMN, FAOD, KET & \\
\hline ACAD9 & 1866 & 18 & 3666 & 611103 & AMN, FAOD, KET & \\
\hline$D E C R 1$ & 1008 & 10 & 2008 & 222745 & AMN, FAOD, KET & \\
\hline ACAT1 & 1284 & 12 & 2484 & 607809 & AMN, FAOD, KET & \\
\hline ACAT2 & 1281 & 9 & 2181 & 100678 & AMN, FAOD, KET & \\
\hline HMGCL & 978 & 9 & 1878 & 613898 & AMN, FAOD, KET & \\
\hline HMGCS2 & 1527 & 10 & 2527 & 600234 & AMN, FAOD, KET & \\
\hline OXCT1 & 1563 & 17 & 3263 & 601424 & AMN, FAOD, KET & \\
\hline G6PC & 1074 & 5 & 1574 & 613742 & $\mathrm{CHO}$ & \\
\hline SLC37A4 & 1356 & 12 & 2556 & 602671 & $\mathrm{CHO}$ & \\
\hline$A G L$ & 4599 & 34 & 7999 & 610860 & $\mathrm{CHO}$ & \\
\hline GBE1 & 2109 & 16 & 3709 & 607839 & $\mathrm{CHO}$ & \\
\hline PYGL & 2544 & 20 & 4544 & 613741 & $\mathrm{CHO}$ & \\
\hline PHKA2 & 3708 & 33 & 7008 & 300798 & $\mathrm{CHO}$ & \\
\hline PHKB & 3282 & 31 & 6382 & 172490 & $\mathrm{CHO}$ & \\
\hline PHKG2 & 1233 & 10 & 2233 & 172471 & $\mathrm{CHO}$ & \\
\hline PHKA1 & 3723 & 33 & 7023 & 311870 & $\mathrm{CHO}$ & \\
\hline PHKG1 & 1260 & 11 & 2360 & 172470 & $\mathrm{CHO}$ & \\
\hline GYS2 & 2112 & 16 & 3712 & 138571 & $\mathrm{CHO}$ & \\
\hline
\end{tabular}




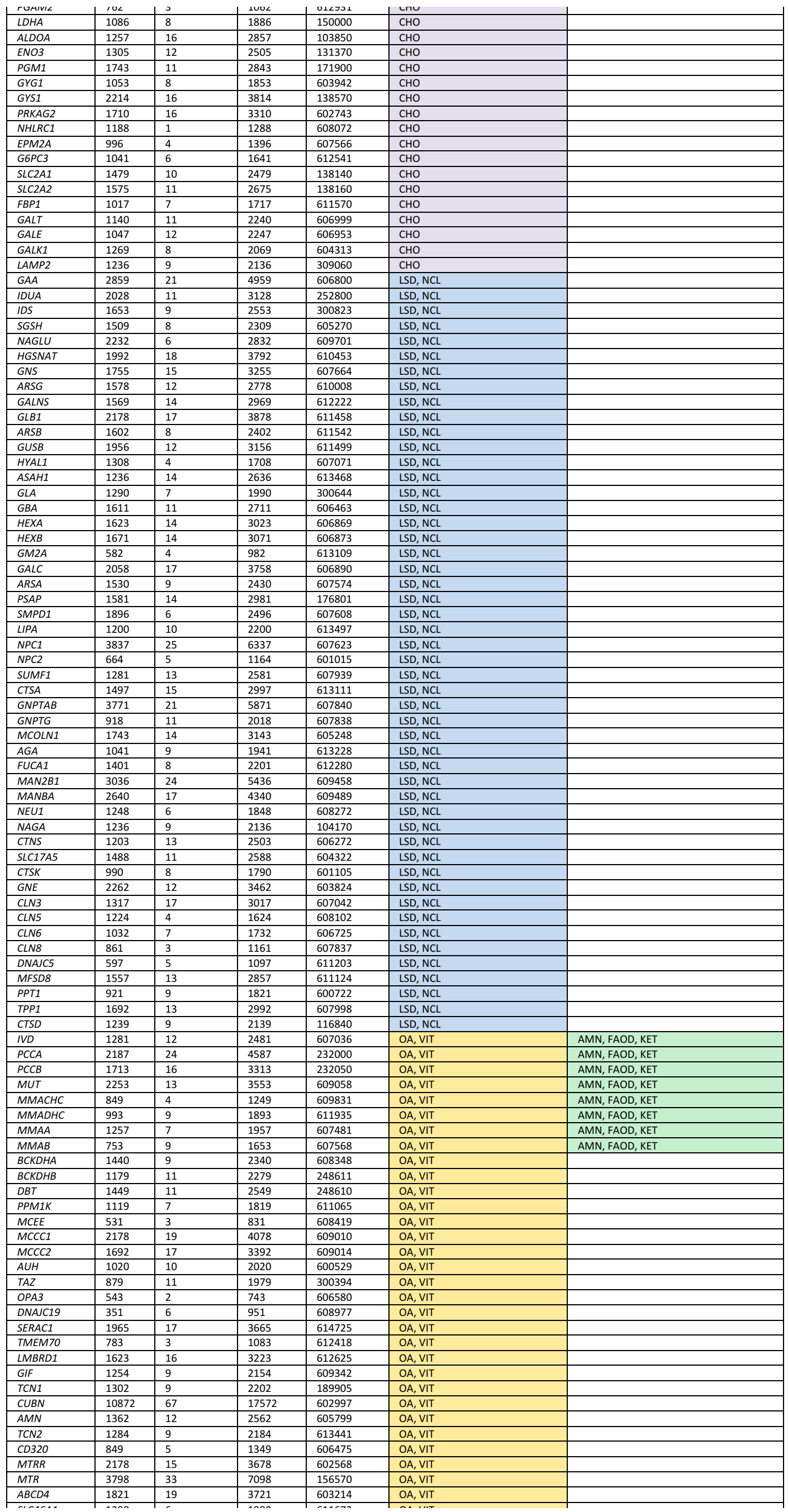




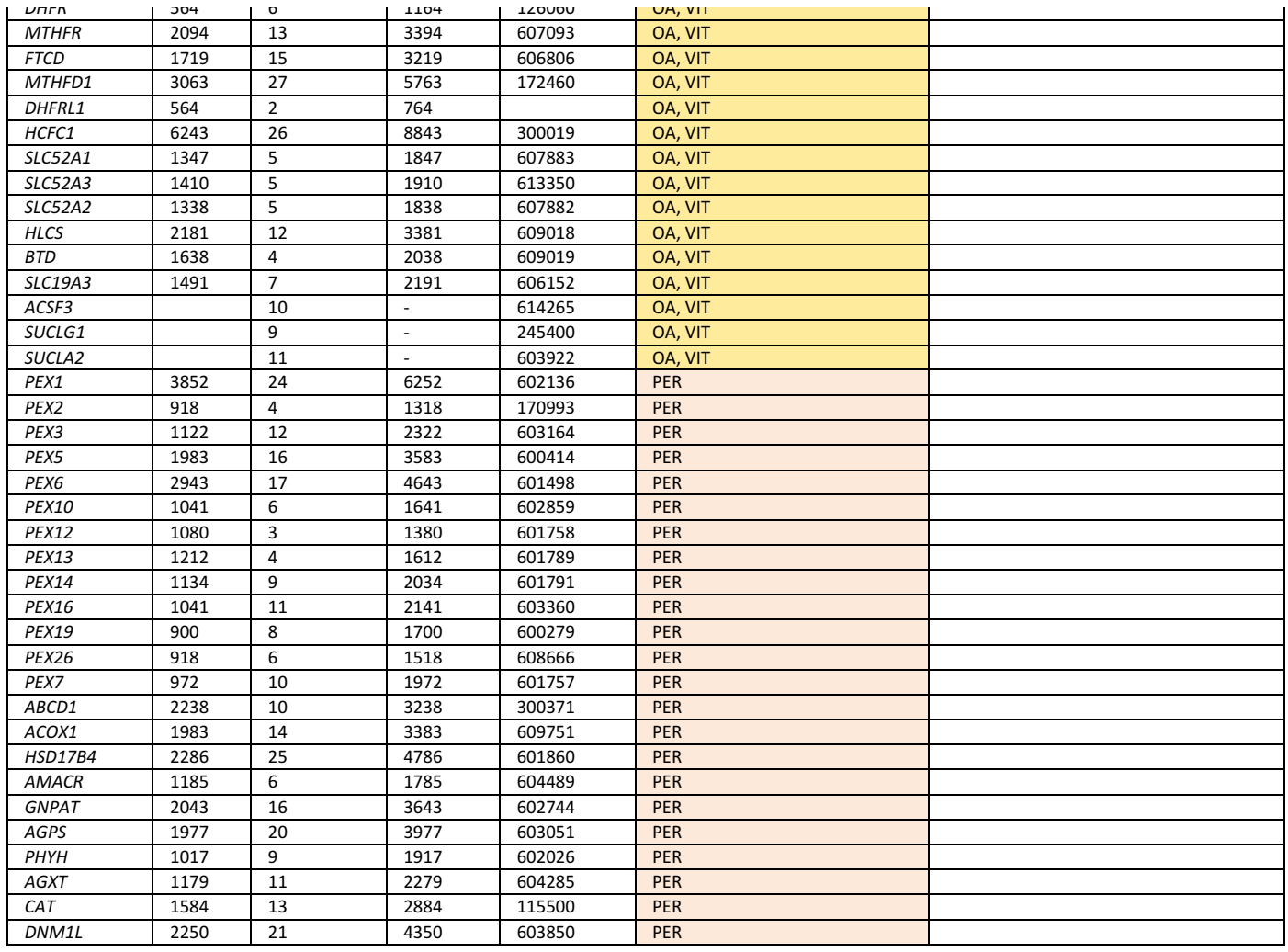




\section{Supplementary Table S3: Details of individuals in whom no primary pathogenic variants were identified}

Results are grouped by sub-panels - (AMN, FAOD, KET), (LSD, NCL), (OA, VIT), (AA, NT), (CHO), (PER), followed by combinations.

Key - AA Disorders of amino acid metabolism including phenylketonuria, and cerebral organic acid disorders; AMN Disorders associated with hyperammonaemia; CHO Disorders of carbohydrate metabolism; FAOD Fatty acid oxidation defects including multiple acyl-CoA dehydrogenase deficiency; KET Disorders of ketogenesis or ketolysis; LSD Lysosomal disorders: includes lysosomal storage disorders, transport defects and protease defects; NCL Neuronal ceroid lipofuscinoses; NT Disorders of neurotransmission (includes pterins, tyrosinaemia); OA Organic acidaemias, including disorders of branched chain amino acid catabolism, 3methylglutaconic acidurias; PER Peroxisomal biogenesis disorders and disorders of single peroxisomal enzymes; VIT Folate and cobalamin defects, also riboflavin transport defects, and biotin-responsive disorders.

^ Denotes invasive or hazardous or lengthy or expensive investigations that were avoided by using the NGS approach.

\begin{tabular}{|c|c|c|c|}
\hline $\begin{array}{l}\text { Subject } \\
\#\end{array}$ & $\begin{array}{l}\text { Age at } \\
\text { investigation }\end{array}$ & Key biochemical and clinical features & $\begin{array}{l}\text { Investigations avoided by NGS } \\
\text { (estimated cost given where specific } \\
\text { investigations anticipated) }\end{array}$ \\
\hline \multicolumn{4}{|c|}{ Single sub-panel tested: (AMN, FAOD, KET) } \\
\hline 52 & $<1$ year & Hyperammonaemia & $\begin{array}{l}\text { ^Sequential mutation analysis of } \\
\text { multiple genes and/or liver biopsy and } \\
\text { enzyme activities in liver tissue }\end{array}$ \\
\hline 53 & $<1$ year & Death at 2 days of age. Suspected FAOD. & $\begin{array}{l}\wedge \text { Sequential mutation analysis of } \\
\text { multiple genes }\end{array}$ \\
\hline 54 & $<1$ year & $\begin{array}{l}\text { Prematurity, severe lactic acidosis and profound } \\
\text { hyperammonaemia with mildly elevated citrulline, normal } \\
\text { arginine. Possible urea cycle disorder or organic } \\
\text { acidaemia. }\end{array}$ & $\begin{array}{l}\wedge \text { Sequential mutation analysis of } \\
\text { multiple genes and/or liver biopsy and } \\
\text { enzyme activities in liver tissue }\end{array}$ \\
\hline 55 & $1-11$ years & $\begin{array}{l}\text { Common acute lymphoblastic leukaemia. Encephalopathy } \\
\text { one week following high dose methotrexate. } \\
\text { Hyperammonaemia with low citrulline and low orotate. } \\
\text { Possible carbamoyl phosphate synthetase (CPS) or N- } \\
\text { acetylglutamate synthase (NAGS) deficiency. }\end{array}$ & $\begin{array}{l}\wedge \text { Sequential mutation analysis of } \\
\text { multiple genes and/or liver biopsy and } \\
\text { enzyme activities in liver tissue }\end{array}$ \\
\hline 56 & $1-11$ years & $\begin{array}{l}\text { Sudden unexplained death in infancy, steatosis in liver } \\
\text { post-mortem }\end{array}$ & $\begin{array}{l}\wedge \text { Sequential mutation analysis of } \\
\text { multiple genes }\end{array}$ \\
\hline 57 & $1-11$ years & Recurrent ketotic hypoglycaemia & $\begin{array}{l}\text { ^Hospital admission, diagnostic fast } \\
\text { with serial glucose, lactate, ketone } \\
\text { analyses and full metabolic/endocrine } \\
\text { profiles }\end{array}$ \\
\hline 58 & $1-11$ years & $\begin{array}{l}\text { Sudden onset of intractable seizures, extensive lipid } \\
\text { accumulation in multiple organs on post-mortem. Low } \\
\text { free carnitine. Suspected carnitine transport defect. }\end{array}$ & $\begin{array}{l}\text { Further clinical and biochemical } \\
\text { phenotyping }\end{array}$ \\
\hline \multicolumn{4}{|c|}{ Single sub-panel tested: (LSD, NCL) } \\
\hline 59 & $1-11$ years & $\begin{array}{l}\text { Developmental regression with myoclonic epilepsy and } \\
\text { cherry red spots. MRI brain normal. Normal lysosomal } \\
\text { enzymes. }\end{array}$ & $\begin{array}{l}\text { Further clinical and biochemical } \\
\text { phenotyping }\end{array}$ \\
\hline 60 & $1-11$ years & $\begin{array}{l}\text { Short stature, coarse features, splenomegaly, } \\
\text { cardiomyopathy, dysostosis multiplex. Failure to thrive } \\
\text { and developmental delay. Elevated urine dermatan } \\
\text { sulfate and heparan sulfate but no MPS disorder revealed } \\
\text { on enzyme testing. }\end{array}$ & $\begin{array}{l}\text { ^Sequential mutation analysis of } \\
\text { multiple genes }\end{array}$ \\
\hline 61 & $12-17$ years & $\begin{array}{l}\text { Joint contractures, autistic spectrum disorders. Mildly } \\
\text { reduced leukocyte alpha-mannosidase activity. Suspected } \\
\text { alpha-mannosidosis. }\end{array}$ & Single gene mutation analysis \\
\hline
\end{tabular}




\begin{tabular}{|c|c|c|c|}
\hline 62 & $12-17$ years & $\begin{array}{l}\text { Cognitive decline with ataxia and abnormal eye } \\
\text { movements. Possible neuronal ceroid lipofuscinosis. }\end{array}$ & $\begin{array}{l}\text { ^Sequential mutation analysis of } \\
\text { multiple genes }\end{array}$ \\
\hline \multicolumn{4}{|c|}{$\begin{array}{c}\text { Single sub-panel tested: }(\mathrm{OA}, \mathrm{VIT}) \\
\end{array}$} \\
\hline 63 & $1-11$ years & $\begin{array}{l}\text { Developmental delay, failure to thrive, feeding difficulties, } \\
\text { gastro-oesophageal reflux, maternal vitamin B12 } \\
\text { deficiency. Low vitamin B12 and methylmalonic aciduria. }\end{array}$ & $\begin{array}{l}{ }^{\wedge} \text { Sequential mutation analysis of } \\
\text { multiple genes and/or skin biopsy and } \\
\text { complementation studies in fibroblasts }\end{array}$ \\
\hline 64 & $<1$ year & Asymptomatic but elevated urinary methylmalonic acid. & $\begin{array}{l}{ }^{\wedge} \text { Sequential mutation analysis of } \\
\text { multiple genes and/or skin biopsy and } \\
\text { complementation studies in fibroblasts }\end{array}$ \\
\hline 65 & $12-17$ years & $\begin{array}{l}\text { Reactive lymphadenopathy, incidental finding of low } \\
\text { vitamin B12 }\end{array}$ & $\begin{array}{l}{ }^{\wedge} \text { Sequential mutation analysis of } \\
\text { multiple genes }\end{array}$ \\
\hline \multicolumn{4}{|c|}{ Single sub-panel tested: (AA, NT) } \\
\hline 66 & $<1$ year & $\begin{array}{l}\text { Previous diagnosis of Beckwith-Wiedemann syndrome } \\
\text { with macroglossia. Upper limb hypotonia, ventricular } \\
\text { septal defect, accessory nipples, thin upper lips. Elevated } \\
\text { 2-hydroxyglutaric acid in urine. }\end{array}$ & $\begin{array}{l}\text { ^Sequential mutation analysis of } \\
\text { multiple genes }\end{array}$ \\
\hline \multicolumn{4}{|c|}{ Single sub-panel tested: (CHO) } \\
\hline 67 & $<1$ year & $\begin{array}{l}\text { Failure to thrive with recurrent lower respiratory tract } \\
\text { infections and pulmonary hypertension. Neutropenia. } \\
\text { Possible G6PC3 deficiency (Dursun syndrome) }\end{array}$ & Single gene mutation analysis \\
\hline 68 & $<1$ year & $\begin{array}{l}\text { Prematurity, frequent desaturations and apnoea. } \\
\text { Hypoglycorrhacia (CSF : plasma glucose ratio <0.5). } \\
\text { Suspected GLUT1 deficiency }\end{array}$ & Single gene mutation analysis \\
\hline 69 & $<1$ year & $\begin{array}{l}\text { Hepatomegaly, mouth ulcers, skin abscesses, recurrent } \\
\text { upper respiratory tract infections, stenosing laryngitis. } \\
\text { Hypoglycaemia with elevated lactate, triglycerides and } \\
\text { urate. Suspected glycogen storage disease 1a or } 1 \mathrm{~b} .\end{array}$ & $\begin{array}{l}\text { ^Sequential mutation analysis of } \\
\text { multiple genes }\end{array}$ \\
\hline \multicolumn{4}{|c|}{ Combination of sub-panels tested: (CHO) (AMN, FAOD, KET) } \\
\hline 70 & $1-11$ years & $\begin{array}{l}\text { Hypoglycaemia with and without ketosis. Global } \\
\text { developmental delay and autism. Suspected FAOD, } \\
\text { ketogenesis defect or glycogen synthase deficiency. }\end{array}$ & $\begin{array}{l}\text { ^Hospital admission, diagnostic fast } \\
\text { with serial glucose, lactate, ketone } \\
\text { analyses and full metabolic/endocrine } \\
\text { profiles }\end{array}$ \\
\hline 71 & $1-11$ years & $\begin{array}{l}\text { Ketotic hypoglycaemia with elevated free fatty acids. } \\
\text { Short stature, congenital heart disease, deafness, } \\
\text { dysmorphism. }\end{array}$ & $\begin{array}{l}\text { ^Hospital admission, diagnostic fast } \\
\text { with serial glucose, lactate, ketone } \\
\text { analyses and full metabolic/endocrine } \\
\text { profiles }\end{array}$ \\
\hline 72 & $1-11$ years & Recurrent hypoglycaemia with severe ketosis. Seizures. & $\begin{array}{l}\text { ^Hospital admission, diagnostic fast } \\
\text { with serial glucose, lactate, ketone } \\
\text { analyses and full metabolic/endocrine } \\
\text { profiles }\end{array}$ \\
\hline 73 & $1-11$ years & Recurrent hypoglycaemia & $\begin{array}{l}\text { ^Hospital admission, diagnostic fast } \\
\text { with serial glucose, lactate, ketone } \\
\text { analyses and full metabolic/endocrine } \\
\text { profiles }\end{array}$ \\
\hline 74 & $1-11$ years & $\begin{array}{l}\text { Recurrent hypoglycaemia with ketoacidosis. Recurrent } \\
\text { febrile seizures. Possible ketogenesis defect. }\end{array}$ & $\begin{array}{l}\text { ^Hospital admission, diagnostic fast } \\
\text { with serial glucose, lactate, ketone } \\
\text { analyses and full metabolic/endocrine } \\
\text { profiles }\end{array}$ \\
\hline 75 & $1-11$ years & Recurrent ketotic hypoglycaemia. Seizures. & $\begin{array}{l}\text { ^Hospital admission, diagnostic fast } \\
\text { with serial glucose, lactate, ketone } \\
\text { analyses and full metabolic/endocrine } \\
\text { profiles }\end{array}$ \\
\hline 76 & $1-11$ years & Recurrent unexplained hypoglycaemia & $\begin{array}{l}\text { ^Hospital admission, diagnostic fast } \\
\text { with serial glucose, lactate, ketone } \\
\text { analyses and full metabolic/endocrine } \\
\text { profiles }\end{array}$ \\
\hline
\end{tabular}




\begin{tabular}{|c|c|c|c|}
\hline 77 & $1-11$ years & Recurrent hypoglycaemia & $\begin{array}{l}\text { ^Hospital admission, diagnostic fast } \\
\text { with serial glucose, lactate, ketone } \\
\text { analyses and full metabolic/endocrine } \\
\text { profiles }\end{array}$ \\
\hline 78 & $<1$ year & $\begin{array}{l}\text { Developmental delay, microcephaly, hepatomegaly, } \\
\text { muscle biopsy suggestive of glycogen storage disease } \\
\text { (GSD VII). Episode of profound hypoglycaemia and } \\
\text { metabolic acidosis. }\end{array}$ & $\begin{array}{l}\wedge \text { Sequential mutation analysis of } \\
\text { multiple genes }\end{array}$ \\
\hline 79 & $1-11$ years & $\begin{array}{l}\text { Episodic weakness and ataxia. Ketoacidosis, } \\
\text { hypoglycaemia and hyperammonaemia with mild } \\
\text { elevation of 3-hydroxybutyrate. Suspected ketolytic } \\
\text { disorder or glycogen storage disorder }\end{array}$ & $\begin{array}{l}\text { ^Hospital admission, diagnostic fast } \\
\text { with serial glucose, lactate, ketone } \\
\text { analyses and full metabolic/endocrine } \\
\text { profiles, or ^sequential mutation } \\
\text { analysis of multiple genes }\end{array}$ \\
\hline 80 & $1-11$ years & $\begin{array}{l}\text { Ketotic hypoglycaemia, gastrointestinal disturbance, joint } \\
\text { laxity, hearing loss. }\end{array}$ & $\begin{array}{l}\text { ^Hospital admission, diagnostic fast } \\
\text { with serial glucose, lactate, ketone } \\
\text { analyses and full metabolic/endocrine } \\
\text { profiles }\end{array}$ \\
\hline 81 & $1-11$ years & Recurrent ketotic hypoglycaemia & $\begin{array}{l}\text { ^Hospital admission, diagnostic fast } \\
\text { with serial glucose, lactate, ketone } \\
\text { analyses and full metabolic/endocrine } \\
\text { profiles }\end{array}$ \\
\hline 82 & $1-11$ years & $\begin{array}{l}\text { Muscle weakness. Muscle biopsy nonspecific. Possible } \\
\text { glycogen storage disorder. }\end{array}$ & $\begin{array}{l}\text { ^Sequential mutation analysis of } \\
\text { multiple genes }\end{array}$ \\
\hline 83 & $<1$ year & $\begin{array}{l}\text { Poor feeding, hypoglycaemia and lactic acidosis. } \\
\text { Cardiorespiratory arrest day } 3 \text { of life leading to hypoxic } \\
\text { brain injury. Glycerol in urine. Suspected fructose 1,6- } \\
\text { bisphosphatase deficiency }\end{array}$ & Single gene mutation analysis \\
\hline 84 & $1-11$ years & $\begin{array}{l}\text { Atypical ketotic hypoglycaemia. Known pancreatic } \\
\text { exocrine insufficiency (aetiology uncertain). Neutropenia. }\end{array}$ & $\begin{array}{l}\text { ^Hospital admission, diagnostic fast } \\
\text { with serial glucose, lactate, ketone } \\
\text { analyses and full metabolic/endocrine } \\
\text { profiles }\end{array}$ \\
\hline \multicolumn{4}{|c|}{ Combination of sub-panels tested: (OA, VIT) (CHO) (AMN, FAOD, KET) } \\
\hline 85 & $1-11$ years & $\begin{array}{l}\text { Recurrent ketotic hypoglycaemia with elevated lactate, } \\
\text { hypotonia. }\end{array}$ & $\begin{array}{l}\text { ^Hospital admission, diagnostic fast } \\
\text { with serial glucose, lactate, ketone } \\
\text { analyses and full metabolic/endocrine } \\
\text { profiles }\end{array}$ \\
\hline 86 & $1-11$ years & $\begin{array}{l}\text { Recurrent hypoglycaemia. Mild developmental delay, } \\
\text { hearing impairment. }\end{array}$ & $\begin{array}{l}\text { ^Hospital admission, diagnostic fast } \\
\text { with serial glucose, lactate, ketone } \\
\text { analyses and full metabolic/endocrine } \\
\text { profiles }\end{array}$ \\
\hline 87 & $1-11$ years & $\begin{array}{l}\text { Two episodes of ketoacidosis, one with elevated lactate } \\
\text { and one with hypoglycaemia. Suspected fructose-1,6- } \\
\text { bisphosphatase deficiency or succinyl-CoA:3-ketoacid CoA } \\
\text { transferase (SCOT) deficiency. }\end{array}$ & $\begin{array}{l}\text { ^Hospital admission, diagnostic fast } \\
\text { with serial glucose, lactate, ketone } \\
\text { analyses and full metabolic/endocrine } \\
\text { profiles, skin biopsy, fibroblast culture } \\
\text { and enzyme analysis }\end{array}$ \\
\hline 88 & $<1$ year & Recurrent hypoglycaemia. Short gut and liver disease. & $\begin{array}{l}\text { ^Hospital admission, diagnostic fast } \\
\text { with serial glucose, lactate, ketone } \\
\text { analyses and full metabolic/endocrine } \\
\text { profiles }\end{array}$ \\
\hline 89 & $1-11$ years & $\begin{array}{l}\text { Recurrent ketotic hypoglycaemia, hypotonia, drooling, } \\
\text { dyspraxia. }\end{array}$ & $\begin{array}{l}\text { ^Hospital admission, diagnostic fast } \\
\text { with serial glucose, lactate, ketone } \\
\text { analyses and full metabolic/endocrine } \\
\text { profiles }\end{array}$ \\
\hline \multicolumn{4}{|c|}{ Combination of sub-panels tested: (OA, VIT) (AA, NT) } \\
\hline 90 & $1-11$ years & Developmental delay, severe vitamin B12 responsive & Single gene mutation analysis \\
\hline
\end{tabular}




\begin{tabular}{|c|c|c|c|}
\hline & & $\begin{array}{l}\text { megaloblastic anaemia, mildly elevated homocysteine but } \\
\text { no methylmalonic acid detected in urine. Suspected } \\
\text { transcobalamin II deficiency. }\end{array}$ & \\
\hline \multicolumn{4}{|c|}{ Combination of subpanels tested: (AA, NT) (AMN, FAOD, KET) } \\
\hline 91 & $<1$ year & $\begin{array}{l}\text { Failure to thrive, anaemia. Elevated methylmalonic acid in } \\
\text { urine and homocysteine in blood. Suspected } \\
\text { remethylation disorder. }\end{array}$ & $\begin{array}{l}\text { ^Sequential mutation analysis of } \\
\text { multiple genes }\end{array}$ \\
\hline 92 & $<1$ year & $\begin{array}{l}\text { Cardiomyopathy, multiple organ failure. Severe } \\
\text { hyperammonaemia and lactic acidosis. }\end{array}$ & $\begin{array}{l}\text { ^Sequential mutation analysis of } \\
\text { multiple genes, or liver biopsy and } \\
\text { enzyme analysis followed by single } \\
\text { gene mutation analysis }\end{array}$ \\
\hline \multicolumn{4}{|c|}{ All sub-panels tested } \\
\hline 93 & $<1$ year & $\begin{array}{l}\text { Respiratory distress, lethargy and poor feeding. Moderate } \\
\text { hyperammonaemia with elevated lactate and ketonuria. } \\
\text { Suspected urea cycle disorder or organic acidaemia. }\end{array}$ & $\begin{array}{l}\text { ^Sequential mutation analysis of } \\
\text { multiple genes and/or liver biopsy and } \\
\text { enzyme activities in liver tissue }\end{array}$ \\
\hline 94 & $1-11$ years & $\begin{array}{l}\text { Megaloblastic anaemia. Suspected cerebral folate } \\
\text { deficiency }\end{array}$ & Single gene mutation analysis \\
\hline 95 & $\begin{array}{l}\text { Adult }(18+ \\
\text { years })\end{array}$ & $\begin{array}{l}\text { Hepatosplenomegaly, chronic thrombocytopenia since } 3 \\
\text { years of age, acute pancytopenia. Bone marrow } \\
\text { examination normal. Lysosomal enzymes normal. } \\
\text { Suspected Gaucher-like disorder. }\end{array}$ & $\begin{array}{l}\text { Further clinical and biochemical } \\
\text { phenotyping }\end{array}$ \\
\hline 96 & $\begin{array}{l}\text { Adult }(18+ \\
\text { years })\end{array}$ & $\begin{array}{l}\text { Known MTHFR homozygous mutation. Epilepsy, autism, } \\
\text { hypermobility, high arched palate. Symptoms since } 3 \\
\text { years of age. }\end{array}$ & $\begin{array}{l}\text { Further clinical and biochemical } \\
\text { phenotyping }\end{array}$ \\
\hline 97 & $12-17$ years & $\begin{array}{l}\text { Muscle weakness, wasting and contractures. Symptoms } \\
\text { since } 5 \text { years of age. }\end{array}$ & $\begin{array}{l}\text { Further clinical and biochemical } \\
\text { phenotyping }\end{array}$ \\
\hline 98 & $1-11$ years & $\begin{array}{l}\text { Ketotic hypoglycaemia, seizure secondary to } \\
\text { hypoglycaemia. }\end{array}$ & $\begin{array}{l}\text { ^Hospital admission, diagnostic fast } \\
\text { with serial glucose, lactate, ketone } \\
\text { analyses and full metabolic/endocrine } \\
\text { profiles }\end{array}$ \\
\hline 99 & $12-17$ years & $\begin{array}{l}\text { Known myotonic dystrophy. Cardiomyopathy since } 22 \\
\text { months, shortness of breath on exertion, hyper-lgM } \\
\text { syndrome, intellectual disability, vasculitis, hypermobility, } \\
\text { lower limb upper motor neurone signs. Abnormal white } \\
\text { matter changes on brain MRI. }\end{array}$ & $\begin{array}{l}\text { Further clinical and biochemical } \\
\text { phenotyping }\end{array}$ \\
\hline 100 & $1-11$ years & $\begin{array}{l}\text { Bilateral optic atrophy, growth retardation, cerebral } \\
\text { calcifications. Macrocytic anaemia. }\end{array}$ & $\begin{array}{l}\text { Further clinical and biochemical } \\
\text { phenotyping }\end{array}$ \\
\hline 101 & $1-11$ years & $\begin{array}{l}\text { Ketotic hypoglycaemia. Suspected FAOD or disorder of } \\
\text { bile acid }\end{array}$ & $\begin{array}{l}\text { ^Hospital admission, diagnostic fast } \\
\text { with serial glucose, lactate, ketone } \\
\text { analyses and full metabolic/endocrine } \\
\text { profiles }\end{array}$ \\
\hline 102 & $<1$ year & $\begin{array}{l}\text { Developmental regression, movement disorder, } \\
\text { peripheral hypertonia, hypercalcaemia (suspected familial } \\
\text { hypocalciuric hypercalcaemia), gastro-oesophageal reflux. } \\
\text { Mildly raised HIAA and HVA levels. Suspected } \\
\text { neurotransmitter disorders, non-ketotic } \\
\text { hyperglycinaemia, serine synthase deficiency, or disorders } \\
\text { of purine metabolism disorders especially HPRT. }\end{array}$ & $\begin{array}{l}\text { ^Sequential mutation analysis of } \\
\text { multiple genes }\end{array}$ \\
\hline
\end{tabular}




\section{Supplementary Table S4: Details of individuals in whom incidental carrier findings were identified}

Results are grouped by sub-panels - (AMN, FAOD, KET), (LSD, NCL), (OA, VIT), (AA, NT), (CHO), (PER), followed by combinations. Grouping of sub-panels is indicated by parenthesis. Only individuals in whom incidental carrier findings were found are reported. Details of individuals in whom a genetic diagnosis was made are provided in Table 1 and details of individuals in whom no primary pathogenic variants were identified are provided in Table S2. Numbers of variants of unknown clinical significance are reported in Table S4.

Key - AA Disorders of amino acid metabolism including phenylketonuria, and cerebral organic acid disorders; AMN Disorders associated with hyperammonaemia; $\mathrm{CHO}$ Disorders of carbohydrate metabolism; FAOD Fatty acid oxidation defects including multiple acyl-CoA dehydrogenase deficiency; KET Disorders of ketogenesis or ketolysis; LSD Lysosomal disorders: includes lysosomal storage disorders, transport defects and protease defects; NCL Neuronal ceroid lipofuscinoses; NT Disorders of neurotransmission (includes pterins, tyrosinaemia); OA Organic acidaemias, including disorders of branched chain amino acid catabolism, 3methylglutaconic acidurias; PER Peroxisomal biogenesis disorders and disorders of single peroxisomal enzymes; VIT Folate and cobalamin defects, also riboflavin transport defects, and biotin-responsive disorders.

\begin{tabular}{|c|c|c|c|c|}
\hline Subject \# & Sub-panels tested & Result & Zygosity & Interpretation \\
\hline 3 & (AMN, FAOD, KET) & ACADM c.250C>T [p.(Leu84Phe)] & heterozygous & Carrier finding \\
\hline 16 & (LSD, NCL) & GAA c.859-3C>G & heterozygous & Carrier finding \\
\hline 20 & $(\mathrm{OA}, \mathrm{VIT})$ & ACSF3 c.1672C>T [p.(Arg588Trp )] & heterozygous & Carrier finding \\
\hline 29 & (AA, NT) & PAH c.688G >A [p.(Val230lle)] & heterozygous & Carrier finding \\
\hline 38 & (PER) & CAT c.1169G>A [p.(Gly390Asp)] & heterozygous & Carrier finding \\
\hline 47 & All sub-panels & ASL c.392C>T [p.(Thr131 Met)] & heterozygous & Carrier finding \\
\hline 48 & All sub-panels & GNMT c.529C>A [p.(His177Asn)] & heterozygous & Carrier finding \\
\hline 51 & All sub-panels & $\begin{array}{l}\text { CUBN c.2594G>A [p.(Ser865Asn)] } \\
\text { HPD c.1005C>G [p.(Ile335Met)] }\end{array}$ & $\begin{array}{l}\text { heterozygous } \\
\text { heterozygous }\end{array}$ & $\begin{array}{l}\text { Carrier finding } \\
\text { Carrier finding }\end{array}$ \\
\hline 52 & (AMN, FAOD, KET) & SLC22A5 c.136C>T [p.(Pro46Ser)] & heterozygous & Carrier finding \\
\hline 67 & $(\mathrm{CHO})$ & $S L C 2 A 2$ c.497-2A>T & heterozygous & Carrier finding \\
\hline 85 & $(\mathrm{OA}, \mathrm{VIT})(\mathrm{CHO})(\mathrm{AMN}, \mathrm{FAO}, \mathrm{KET})$ & $P C C B$ c.1550C $>\mathrm{T}$ [p.(Ala517Val)] & heterozygous & Carrier finding \\
\hline 86 & $(\mathrm{OA}, \mathrm{VIT})(\mathrm{CHO})(\mathrm{AMN}, \mathrm{FAO}, \mathrm{KET})$ & MMAB c.12C>A [p.(Cys4Ter)] & heterozygous & Carrier finding \\
\hline 93 & All sub-panels & $\begin{array}{l}\text { MUT c.1106G>A [p.(Arg369His)] } \\
\text { NAGS c.785G>C [p.(Gly262Ala)] } \\
\text { GUSB c.1121G>T [p.(Arg374Leu)] }\end{array}$ & $\begin{array}{l}\text { heterozygous } \\
\text { heterozygous } \\
\text { heterozygous }\end{array}$ & $\begin{array}{l}\text { Carrier finding } \\
\text { Carrier finding } \\
\text { Carrier finding }\end{array}$ \\
\hline 94 & All sub-panels & $\begin{array}{l}\text { GALT c.563A>G [p. (Gln188Arg)] } \\
\text { CLN8 c. 806A>T [p. (Glu269Val)] }\end{array}$ & $\begin{array}{l}\text { heterozygous } \\
\text { heterozygous }\end{array}$ & $\begin{array}{l}\text { Carrier finding } \\
\text { Carrier finding }\end{array}$ \\
\hline 95 & All sub-panels & PEX12 c.102A>T [p. (Arg34Ser)] & heterozygous & Carrier finding \\
\hline 96 & All sub-panels & $\begin{array}{l}\text { ACSF3 c.1672C>T [p.(Arg558Trp)] } \\
\text { MTHFR c.665C>T [p.(Ala222Val)] }\end{array}$ & $\begin{array}{l}\text { heterozygous } \\
\text { homozygous }\end{array}$ & $\begin{array}{l}\text { Carrier finding } \\
\text { Known diagnosis }\end{array}$ \\
\hline 97 & All sub-panels & GNE c.2179G >T [p.(Val727Leu)] & heterozygous & Carrier finding \\
\hline 98 & All sub-panels & $\begin{array}{l}\text { MMAB c.394T>C [p.(Cys132Arg)] } \\
\text { HEXA c.745C>T [p.(Arg249Trp)] }\end{array}$ & $\begin{array}{l}\text { heterozygous } \\
\text { heterozygous }\end{array}$ & $\begin{array}{l}\text { Carrier finding } \\
\text { Carrier finding }\end{array}$ \\
\hline
\end{tabular}




\section{Supplementary Table S5: Number of variants of unknown clinical significance (VUS)}

Results are grouped by sub-panels - (AMN, FAOD, KET), (LSD, NCL), (OA, VIT), (AA, NT), (CHO), (PER), followed by combinations. Grouping of sub-panels is indicated by parenthesis.

Key - AA Disorders of amino acid metabolism including phenylketonuria, and cerebral organic acid disorders; AMM Disorders associated with hyperammonaemia; CHO Disorders of carbohydrate metabolism; FAOD Fatty acid oxidation defects including multiple acyl-CoA dehydrogenase deficiency; KET Disorders of ketogenesis or ketolysis; LSD Lysosomal disorders: includes lysosomal storage disorders, transport defects and protease defects; NCL Neuronal ceroid lipofuscinoses; NT Disorders of neurotransmission (includes pterins, tyrosinaemia); OA Organic acidaemias, including disorders of branched chain amino acid catabolism, 3methylglutaconic acidurias; PER Peroxisomal biogenesis disorders and disorders of single peroxisomal enzymes; VIT Folate and cobalamin defects, also riboflavin transport defects, and biotin-responsive disorders.

\begin{tabular}{|c|c|c|c|}
\hline Sub-panels tested & $\begin{array}{l}\text { Total number of } \\
\text { genes tested }\end{array}$ & $\begin{array}{l}\text { Number of } \\
\text { individuals with VUS } \\
\text { (\% of individuals } \\
\text { tested) }\end{array}$ & $\begin{array}{l}\text { Median number of } \\
\text { VUS per individual } \\
\text { (range) }\end{array}$ \\
\hline (AMN, FAOD, KET) & 28 & $12(71 \%)$ & $1(0-8)$ \\
\hline$(\mathrm{LSD}, \mathrm{NCL})$ & 50 & $12(92 \%)$ & $6(0-15)$ \\
\hline$(\mathrm{OA}, \mathrm{VIT})$ & 50 & $8(80 \%)$ & $2(0-12)$ \\
\hline$(\mathrm{AA}, \mathrm{NT})$ & 43 & $7(78 \%)$ & $1(0-4)$ \\
\hline$(\mathrm{CHO})$ & 32 & $4(80 \%)$ & $5(0-7)$ \\
\hline (PER) & 23 & $2(50 \%)$ & $0.5(0-2)$ \\
\hline (CHO), (AMN, FAOD, KET) & 60 & $15(88 \%)$ & $2(0-4)$ \\
\hline $\begin{array}{l}\text { All sub-panels: (OA, VIT), (CHO), (AA, } \\
\text { NT), (AMN, FAOD, KET), (PER), (LSD, } \\
\text { NCL) }\end{array}$ & 226 & $14(93 \%)$ & $12(0-24)$ \\
\hline$(\mathrm{OA}, \mathrm{VIT}),(\mathrm{CHO}),(\mathrm{AMN}, \mathrm{FAOD}, \mathrm{KET})$ & 110 & $6(100 \%)$ & $8.5(2-17)$ \\
\hline$(\mathrm{OA}, \mathrm{VIT}),(\mathrm{AA}, \mathrm{NT})$ & 93 & $2(100 \%)$ & $10(7-13)$ \\
\hline $\begin{array}{l}\text { (OA, VIT), (CHO), (AA, NT), (AMN, FAOD, } \\
\text { KET) }\end{array}$ & 153 & $1(100 \%)$ & 2 \\
\hline (OA, VIT), (AA, NT), (AMN, FAOD, KET) & 121 & $1(100 \%)$ & 5 \\
\hline$(\mathrm{AA}, \mathrm{NT}),(\mathrm{AMN}, \mathrm{FAOD}, \mathrm{KET})$ & 71 & $1(100 \%)$ & 6 \\
\hline (OA, VIT), (AMN, FAOD, KET) & 78 & $1(50 \%)$ & 4 \\
\hline
\end{tabular}


Supplementary Table S6: Comparison of NGS costs and estimated costs of investigations avoided by NGS. NGS costs based on $£ 950+£ 50$ for each additional panel requested. 'Pre-NGS estimated cost' refers to estimated cost of additional investigations avoided by NGS approach. Where no pathogenic variants were identified, this refers to investigations that would have been undertaken had NGS not been available, and was only estimated where specific investigations could be anticipated e.g. single gene mutation analysis or specific biochemical tests.

\begin{tabular}{|l|c|c|c|}
\hline Category & $\begin{array}{l}\text { Number of } \\
\text { patients }\end{array}$ & NGS cost & $\begin{array}{l}\text { Pre-NGS } \\
\text { estimated cost }\end{array}$ \\
\hline $\begin{array}{l}\text { Primary } \\
\text { pathogenic } \\
\text { variants identified }\end{array}$ & 51 & $£ 47,550$ & $£ 48,900$ \\
\hline $\begin{array}{l}\text { No primary } \\
\text { pathogenic } \\
\text { variants identified }\end{array}$ & 51 & $£ 49,800$ & $£ 32,500$ \\
\hline
\end{tabular}




\section{Supplementary Figure S1: Summary of studies of use of NGS panels in IEM}

Diagnostic yield of studies investigating NGS use in diagnosing IEMs, showing diagnostic yield (\%), number of genes tested, and number of individuals tested. A targeted NGS panel approach was used in the Pupavac, Jones, Yubero and Stranneheim studies. Targeted exome sequencing was used in the Lieber study. Whole exome sequencing was used in the Taylor, Stitziel and Tarailo-Graovac studies. A two-step approach was used in the Legati study (targeted NGS proceeding to WES if no diagnosis) and in the Wortmann study (WES virtual panel proceeding to WES if no diagnosis).

Key - GSDs glycogen storage disorders, CDGs congenital disorders of glycosylation, WES whole exome sequencing

\section{Number of individuals QDiagnostic yield (\%)}

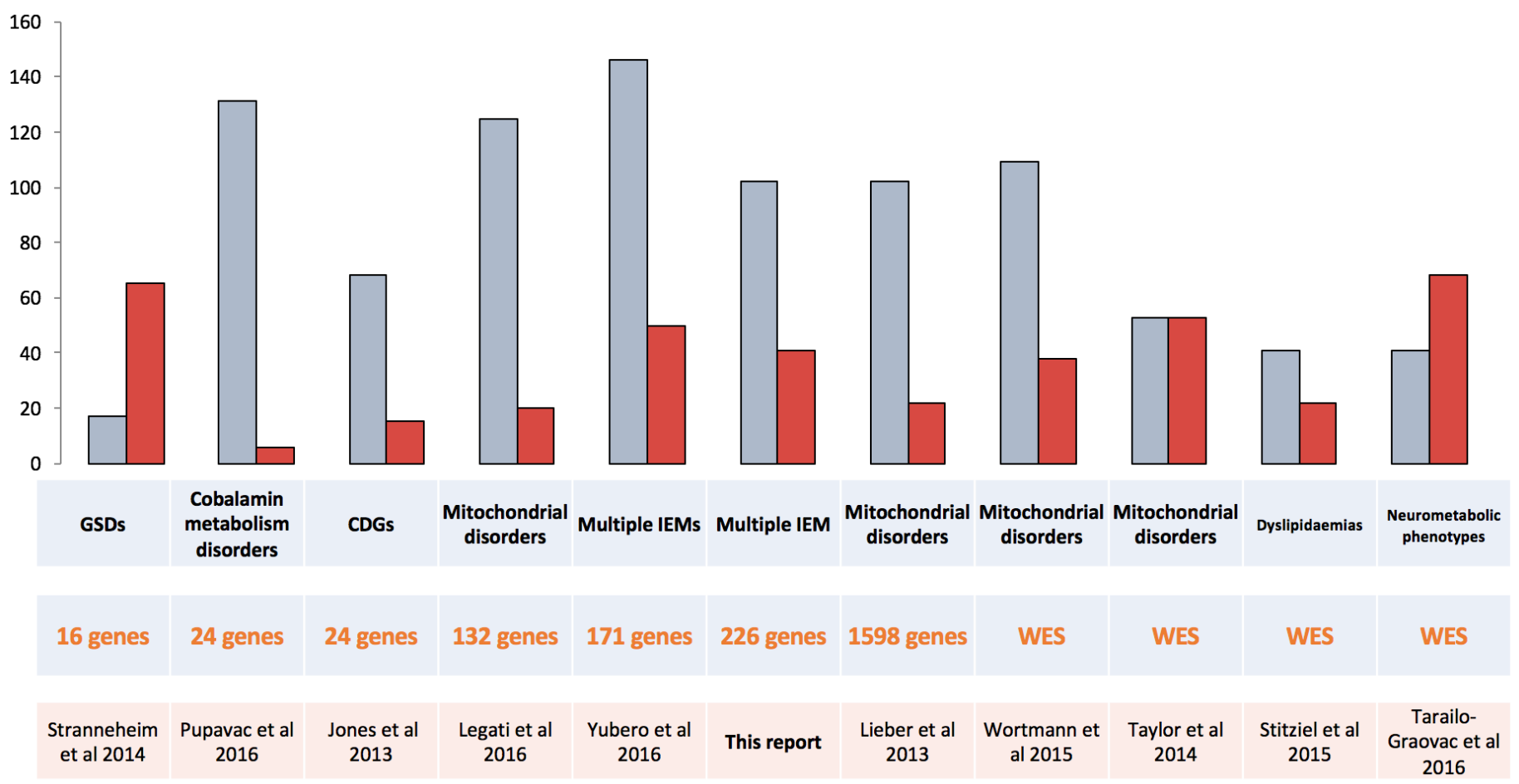




\section{References}

1. Atwal PS. Mutations in the Complex III Assembly Factor Tetratricopeptide 19 Gene TTC19 Are a Rare Cause of Leigh Syndrome. JIMD reports. 2014;14:43-45.

2. Boycott KM, Beaulieu CL, Kernohan KD, et al. Autosomal-Recessive Intellectual Disability with Cerebellar Atrophy Syndrome Caused by Mutation of the Manganese and Zinc Transporter Gene SLC39A8. American journal of human genetics. 2015;97(6):886-893.

3. Castro-Gago M, Dacruz-Alvarez D, Pintos-Martinez E, et al. Exome sequencing identifies a CHKB mutation in Spanish patient with megaconial congenital muscular dystrophy and mtDNA depletion. European journal of paediatric neurology : EJPN : official journal of the European Paediatric Neurology Society. 2014;18(6):796-800.

4. Gai X, Ghezzi D, Johnson MA, et al. Mutations in FBXL4, encoding a mitochondrial protein, cause early-onset mitochondrial encephalomyopathy. American journal of human genetics. 2013;93(3):482-495.

5. Imagawa $\mathrm{E}$, Osaka H, Yamashita A, et al. A hemizygous GYG2 mutation and Leigh syndrome: a possible link? Human genetics. 2014;133(2):225-234.

6. Invernizzi F, Tigano M, Dallabona C, et al. A homozygous mutation in LYRM7/MZM1L associated with early onset encephalopathy, lactic acidosis, and severe reduction of mitochondrial complex III activity. Human mutation. 2013;34(12):1619-1622.

7. Jansen JC, Cirak S, van Scherpenzeel M, et al. CCDC115 Deficiency Causes a Disorder of Golgi Homeostasis with Abnormal Protein Glycosylation. American journal of human genetics. 2016;98(2):310-321.

8. Jansen JC, Timal S, van Scherpenzeel M, et al. TMEM199 Deficiency Is a Disorder of Golgi Homeostasis Characterized by Elevated Aminotransferases, Alkaline Phosphatase, and Cholesterol and Abnormal Glycosylation. American journal of human genetics. 2016;98(2):322-330.

9. Jones MA, Rhodenizer D, da Silva C, et al. Molecular diagnostic testing for congenital disorders of glycosylation (CDG): detection rate for single gene testing and next generation sequencing panel testing. Mol Genet Metab. 2013;110(1-2):78-85.

10. Kodera $\mathrm{H}$, Nakamura $\mathrm{K}$, Osaka $\mathrm{H}$, et al. De novo mutations in SLC35A2 encoding a UDPgalactose transporter cause early-onset epileptic encephalopathy. Human mutation. 2013;34(12):1708-1714.

11. Legati A, Reyes A, Nasca A, et al. New genes and pathomechanisms in mitochondrial disorders unraveled by NGS technologies. Biochimica et biophysica acta. 2016.

12. Lieber DS, Calvo SE, Shanahan K, et al. Targeted exome sequencing of suspected mitochondrial disorders. Neurology. 2013;80(19):1762-1770.5.

13. Lim SC, Smith KR, Stroud DA, et al. A founder mutation in PET100 causes isolated complex IV deficiency in Lebanese individuals with Leigh syndrome. American journal of human genetics. 2014;94(2):209-222.

14. Losfeld ME, Ng BG, Kircher M, et al. A new congenital disorder of glycosylation caused by a mutation in SSR4, the signal sequence receptor 4 protein of the TRAP complex. Human molecular genetics. 2014;23(6):1602-1605.

15. Mohamed M, Ashikov A, Guillard M, et al. Intellectual disability and bleeding diathesis due to deficient CMP--sialic acid transport. Neurology. 2013;81(7):681-687.

16. Ng BG, Wolfe LA, Ichikawa $M$, et al. Biallelic mutations in CAD, impair de novo pyrimidine biosynthesis and decrease glycosylation precursors. Human molecular genetics. 2015;24(11):30503057.

17. Park EJ, Grabinska KA, Guan Z, et al. Mutation of Nogo-B receptor, a subunit of cisprenyltransferase, causes a congenital disorder of glycosylation. Cell metabolism. 2014;20(3):448457. 
18. Pitceathly RD, Rahman S, Wedatilake $\mathrm{Y}$, et al. NDUFA4 mutations underlie dysfunction of a cytochrome c oxidase subunit linked to human neurological disease. Cell reports. 2013;3(6):17951805.

19. Pupavac $\mathrm{M}$, Tian X, Chu J, et al. Added value of next generation gene panel analysis for patients with elevated methylmalonic acid and no clinical diagnosis following functional studies of vitamin B12 metabolism. Mol Genet Metab. 2016;117(3):363-368.

20. Sanchez-Caballero L, Ruzzenente B, Bianchi L, et al. Mutations in Complex I Assembly Factor TMEM126B Result in Muscle Weakness and Isolated Complex I Deficiency. American journal of human genetics. 2016;99(1):208-216.

21. Shamseldin HE, Smith LL, Kentab A, et al. Mutation of the mitochondrial carrier SLC25A42 causes a novel form of mitochondrial myopathy in humans. Human genetics. 2016;135(1):21-30.

22. Shrimal S, Ng BG, Losfeld ME, Gilmore R, Freeze HH. Mutations in STT3A and STT3B cause two congenital disorders of glycosylation. Human molecular genetics. 2013;22(22):4638-4645.

23. Sofou K, Kollberg G, Holmstrom M, et al. Whole exome sequencing reveals mutations in NARS2 and PARS2, encoding the mitochondrial asparaginyl-tRNA synthetase and prolyl-tRNA synthetase, in patients with Alpers syndrome. Molecular genetics \& genomic medicine. 2015;3(1):5968.

24. Spiegel R, Shaag A, Shalev S, Elpeleg O. Homozygous mutation in the APOA1BP is associated with a lethal infantile leukoencephalopathy. Neurogenetics. 2016;17(3):187-190.

25. Stitziel NO, Peloso GM, Abifadel M, et al. Exome sequencing in suspected monogenic dyslipidemias. Circulation. Cardiovascular genetics. 2015;8(2):343-350.

26. Stranneheim $\mathrm{H}$, Engvall $\mathrm{M}$, Naess $\mathrm{K}$, et al. Rapid pulsed whole genome sequencing for comprehensive acute diagnostics of inborn errors of metabolism. BMC genomics. 2014;15:1090.

27. Tarailo-Graovac M, Shyr C, Ross CJ, et al. Exome Sequencing and the Management of Neurometabolic Disorders. New England Journal of Medicine. 2016;374(23):2246-2255.

28. Taylor RW, Pyle A, Griffin $\mathrm{H}$, et al. Use of whole-exome sequencing to determine the genetic basis of multiple mitochondrial respiratory chain complex deficiencies. Jama. 2014;312(1):68-77.

29. Valencia CA, Ankala A, Rhodenizer D, et al. Comprehensive mutation analysis for congenital muscular dystrophy: a clinical PCR-based enrichment and next-generation sequencing panel. PloS one. 2013;8(1):e53083.

30. van Rahden VA, Fernandez-Vizarra E, Alawi M, et al. Mutations in NDUFB11, encoding a complex I component of the mitochondrial respiratory chain, cause microphthalmia with linear skin defects syndrome. American journal of human genetics. 2015;96(4):640-650.

31. Van Scherpenzeel M, Timal S, Rymen D, et al. Diagnostic serum glycosylation profile in patients with intellectual disability as a result of MAN1B1 deficiency. Brain : a journal of neurology. 2014;137(Pt 4):1030-1038.

32. Wortmann SB, Zietkiewicz S, Kousi M, et al. CLPB mutations cause 3-methylglutaconic aciduria, progressive brain atrophy, intellectual disability, congenital neutropenia, cataracts, movement disorder. American journal of human genetics. 2015;96(2):245-257.

33. Wortmann SB, Koolen DA, Smeitink JA, van den Heuvel L, Rodenburg RJ. Whole exome sequencing of suspected mitochondrial patients in clinical practice. J Inherit Metab Dis. 2015;38(3):437-443.

34. Zeevaert R, de Zegher F, Sturiale L, et al. Bone Dysplasia as a Key Feature in Three Patients with a Novel Congenital Disorder of Glycosylation (CDG) Type II Due to a Deep Intronic Splice Mutation in TMEM165. JIMD reports. 2013;8:145-152.

35. Zhang Y, Yu X, Ichikawa M, et al. Autosomal recessive phosphoglucomutase 3 (PGM3) mutations link glycosylation defects to atopy, immune deficiency, autoimmunity, and neurocognitive impairment. The Journal of allergy and clinical immunology. 2014;133(5):1400-1409, 1409.e14011405. 